\title{
Simultaneous feature selection and Support Vector Machine optimisation using the Grasshopper Optimisation Algorithm
}

\author{
Ibrahim Aljarah - Ala' M. Al-Zoubi - Hossam Faris - Mohammad A. \\ Hassonah · Seyedali Mirjalili · Heba Saadeh
}

Received: date / Accepted: date

\begin{abstract}
Background/ introduction: Support Vector Machine (SVM) is considered to be one of the most powerful learning algorithms and is used for a wide range of real world applications. The efficiency of SVM algorithm and its performance mainly depends on the kernel type and its parameters. Furthermore, the feature subset selection that is used to train the SVM model is another important factor that has a major influence on it classification accuracy. The feature subset selection is a very important step in machine learning, specially when dealing with high dimensional data sets. Most of the previous researches handled these important factors separately. Methods: In this paper, we propose a hybrid approach based on the Grasshopper Optimisation Algorithm (GOA), which is a recent algorithm inspired by the biological behaviour shown in swarms of grasshoppers. The goal of the proposed approach is to optimise the parameters of the SVM model, and locate the best features subset simultaneously. Re-
\end{abstract}

(凶) I. Aljarah · A. M. Al-Zoubi · H. Faris - M. A. Hassonah, Business Information Technology Department

King Abdullah II School for Information Technology, The

University of Jordan, Amman, Jordan

E-mail: i.aljarah@ju.edu.jo E-mail: alaah14@gmail.com

E-mail: hossam.faris@ju.edu.jo

E-mail: mohammad.a.hassonah@gmail.com

S. Mirjalili

School of Information and Communication Technology, Griffith University, Nathan, Brisbane, QLD 4111, Australia

E-mail: seyedali.mirjalili@griffithuni.edu.au

Heba Saadeh

Computer Science Department

King Abdullah II School for Information Technology, The

University of Jordan, Amman, Jordan

E-mail: heba.saadeh@ju.edu.jo sults and Conclusions: Eighteen low and high dimensional benchmark data sets are used to evaluate the accuracy of the proposed approach. For verification, the proposed approach is compared with seven well-regarded algorithms. Furthermore, the proposed approach is compared with grid search, which is the most popular technique for tuning SVM parameters. The experimental results show that the proposed approach outperforms all of the other techniques in most of the data sets in terms of classification accuracy, while minimizing the number of selected features.

Keywords SVM · Support vector machine · Grasshopper Optimisation Algorithm - GOA · Optimisation ·

Feature selection $\cdot$ Metaheuristics

\section{Introduction}

Bio-inspired systems have been intensively investigated for optimising different cognitive and learning algorithms $[46,13,23,1]$. One of these algorithms is the Support Vector Machine (SVM), which is a well-known supervised classification algorithm. SVM was first proposed and implemented by Vladimir Vapnik $[42,43]$. The SVM algorithm is one of the most popular supervised models and is considered to be one of the strongest method in the machine learning field. SVM has some powerful characteristics compared to other methods such as excellent generalization performance, which is able to generate high quality decision boundaries based on a small subset of training data points. In addition, the SVM has a high ability to model complex and nonlinear relations $[39,48]$.

The basic idea behind the SVM algorithm is to find the optimal hyperplane that separates two classes by 
maximizing distance between the the margin of the hyperplane and the data points in the given data set.

Due to these capabilities, the SVM has been applied on various applications and shown efficient performance in a wide range of challenging real-world problems, such as bio-medical applications [38,3], pattern recognition [55], anomaly detection $[29,36]$, and many others [49, $54,56,2,31,35,32]$.

Although the SVM algorithm has many advantages, it also suffered from some limitations such as sensitivity to the initial values of its parameters. These parameters include the Cost $(C)$ and the kernel parameters such as the gamma $(\gamma)$ parameter in the Radial Basis Function (RBF) kernel. Improper selection of these parameters can negatively impact the generalization performance of the SVM. In addition to this limitation, SVM is like many other machine learning algorithms, its performance depends on the selected data set features, which is critically important in improving the generalization performance, increasing the computational efficiency and reducing running time, and produce very accurate classification model [28,44].

Grasshopper Optimisation Algorithm (GOA) is a recent metaheuristic optimiser, which was proposed by Saremi et al in [34,27]. GOA mimics the behaviour of grasshopper swarms in nature and simulates the repulsion and attraction forces between the grasshoppers. The authors in [34] showed that this algorithm can outperform other well-regarded and modern metaheuristic algorithms based on a suite of complex benchmark functions and engineering problems.

In this work, we propose a new GOA-SVM model, which applies GOA, for the first time in combination with SVM. In this mode, GOA is utilized for performing feature selection and optimising the parameters of SVM simultaneously. The goal of the model is to maximize the classification accuracy of SVM with the smallest possible number of features. The proposed approach is evaluated based on eighteen public data sets of low and high dimensions. For verification, the proposed approach is compared with seven well regraded and modern algorithms such as Genetic algorithm (GA), Particle Swarm Optimization (PSO), Grey Wolf Optimizer (GWO), Firefly Algorithm (FF), Bat Algorithm (BA), Cuckoo Search (CS) and Multi-Verse Optimiser (MVO). Furthermore, the proposed approach is compared with grid search, which is a classical technique used for tuning SVM parameters.

This paper is organized as follows: In Section 2 we review the previous related works. Section 3 briefly describes the GOA algorithm. Section 4 presents the SVM classifier. Section 5 discusses in detail the proposed approach for feature selection and SVM optimisation based on the GOA algorithm. The experiments and results are implemented and analysed in Section 6. Finally, we summarize the conclusions and future directions of this work in Section 7.

\section{Related Works}

In the literature there have been different trends followed by researchers for tuning the parameters of SVM algorithm. Some researchers simply try different sets of values to tune these parameters by trial and error to reach some acceptable performance. Another traditional approach is to use a simple grid search, but this method is very slow and does not provide satisfactory results due to the large number of parameter combinations [37,57]. A third approach is based on utilizing evolutionary or swarm intelligence algorithms. These algorithms belong to the metaheuristic search algorithms family. They are inspired by some theories and phenomena in nature like biological evolution or the movements of swarms of creatures in nature. Examples of such algorithms which were applied for optimising SVM parameters are Genetic Algorithms (GA) [33], Particle Swarm Optimisation (PSO) [4,6], BAT Algorithm (BA) [41], FireFly (FF) [40], Cuckoo Search (CS) [30], and Grey Wolf Optimiser (GWO) [11].

Realizing the importance of the feature selection process on the classification accuracy of the SVM models, a distinguished line of research extended the application of evolutionary and swarm intelligence algorithms to perform feature selection concurrently with optimising the parameters of the SVM. One of the earliest works that followed this approach was conducted by Huang and Wang in [16]. The authors proposed a GA-based approach for optimising the kernel parameters of the SVM and feature selection with the goal of preserving the classification accuracy of the developed SVM model. Their experimental results showed that GA can outperform the grid search method with even fewer features. Another notable work was conducted by Lin et al in [22]. They used PSO for the same task and showed that it can outperform grid search, however it had very similar performance to GA. Other more recent examples of this approach can be found in $[12,15,20,8$, 24]. Although this line of research is very promising, the amount of work that address optimising the SVM parameters at the same time as performing feature selection are still relatively scarce.

The No Free Lunch theorem in optimisation [45] states that there is and will never be an optimisation algorithm to solve all optimisation problems. Therefore, new algorithms have the potential to outperform the existing ones on a set of problems. The GOA algorithm 
is a newcomer and potentially able to show better results with or without modification as compared to the existing algorithms. Saremi et al. [34] showed that this algorithm outperformed most of the existing algorithms on challenging problems with a large number of local solutions (multi-modal). This justifies the use of this algorithm in this work since we intend to take advantage of its new operators to solve classification problems.

\section{The Grasshopper Optimisation Algorithm}

The GOA algorithm was proposed in 2016 by Saremi et al. [34]. This algorithm mimics the swarming behaviour of grasshoppers in nature. The flying path of a grasshopper in a swarm is affected by three components: social interaction $\left(S_{i}\right)$, gravity $\left(G_{i}\right)$, and wind advection $\left(A_{i}\right)$. In the GOA algorithm, the social interaction is the main mechanism of search defined as follows:

$S_{i}=\sum_{j=1, j \neq i}^{N} s\left(d_{i j}\right) \hat{d_{i j}}$

where $d_{i j}$ is the distance between $i-t h$ and $j-t h$ grasshopper and it is calculated as $d_{i j}=\left|x_{j}-x_{i}\right|, s$ is a function to define the strength of social forces, and $\hat{d_{i j}}=\frac{x_{j}=x_{i}}{d_{i j}}$ is a unit vector from $i-t h$ grasshopper to the $j-t h$ grasshopper.

It may be seen in this equation that the main component of the social interaction is the function $s$. This function defines the movement direction of a grasshopper in the swarm and defined as follows:

$s(r)=f e^{\frac{-r}{l}}-e^{-r}$

where $f$ indicates the intensity of attraction and $l$ is the attractive length scale.

This function causes two types of forces between the grasshoppers: attraction and repulsion. When the distance between two grasshoppers is between [0,2.079], they repel each other to avoid collision. The attraction force increases when the distance is in $[2.079,4]$ to maintain the cohesion of the swarm. When the distance is exactly 2.079, there is no force, and this area is called comfort zone. A conceptual model of attraction, repulsion, and comfort zone is visualized in Fig. 1.

If the distance becomes equal to 2.079 , there is no attraction and repulsion. The attraction force increases from 2.079 unit of distance to nearly 4 and then it gradually decreases. Changing the parameters of the equation for the function $s$ ( $l$ and $f$ ) changes the swarming behaviour significantly. To show the interaction between grasshoppers with respect to comfort area, Fig. 1 shows a conceptual schematic.
The swarm model is efficient in simulating the interactions between grasshoppers. However, it must be adjusted to design an optimisation algorithm. Saremi et al. [34] proposed the following mathematical model search while grasshoppers are interacting. The mathematical model is represented by the following equation:

$X_{i}^{d}=c\left(\sum_{j=1, j \neq i}^{N} c \frac{u b_{d}-l b_{d}}{s} s\left(\left|x_{j}^{d}-x_{i}^{d}\right|\right) \frac{x_{j}-x_{i}}{d_{i j}}\right)+\hat{T}_{d}$

where $u b_{d}$ is the upper bound in the $d-t h$ dimension, $l b_{d}$ is the lower bound in the $d-t h$ dimension, $\hat{T}_{d}$ is the value of $d-t h$ dimension in the target (best solution found so far), and $c$ is a decreasing coefficient to shrink the comfort area, repulsion area, and attraction area.

It may be seen in this equation that the swarm updates the position around a target $\left(\hat{T}_{d}\right)$. The parameter c converges the swarm towards the target. In the GOA algorithm, it is assumed that the target is the best solution obtained so far. While grasshoppers are interacting and chasing the target, the best solution is updated if a better solution is encountered.

The parameter $c$ is the main controlling parameter in the GOA algorithm and, is updated using the following equation:

$c=c_{\max }-l \frac{c_{\max }-c_{\min }}{L}$

where $L$ shows the maximum number of iteration, $l$ is the current iteration, $c_{\max }=1$, and $c_{\min }=0.00001$.

Fig. 2 illustrates the effect of the previous equation. This shows the position of the target in 3D space over 100 iterations of the algorithm. The figure illustrates how the mathematical model is employed to implement the movement of a swarm around a target in a 3D space. Due to the use of vectors in the equations, this can be extended to any number of dimensions.

It has been proven that the GOA is very efficient in finding optimal solutions for challenging problems. Despite the simplicity, the algorithm properly gravitates solutions towards to best regions of the search space. The initial population is very important in GAO since the number of random components is very limited in this algorithm.

The computational complexity of the GOA algorithm (excluding SVM in the proposed algorithm) is of $O(t * d * n * n)$ where $\mathrm{n}$ is the number of solutions, $t$ is the number of iterations, and $d$ is the number of variables. This is because, the distance of a grasshopper to all the others should be calculated in each dimension over the course of iterations. Note that this computational complexity does not consider the cost of objective function since it varies for every problem. 


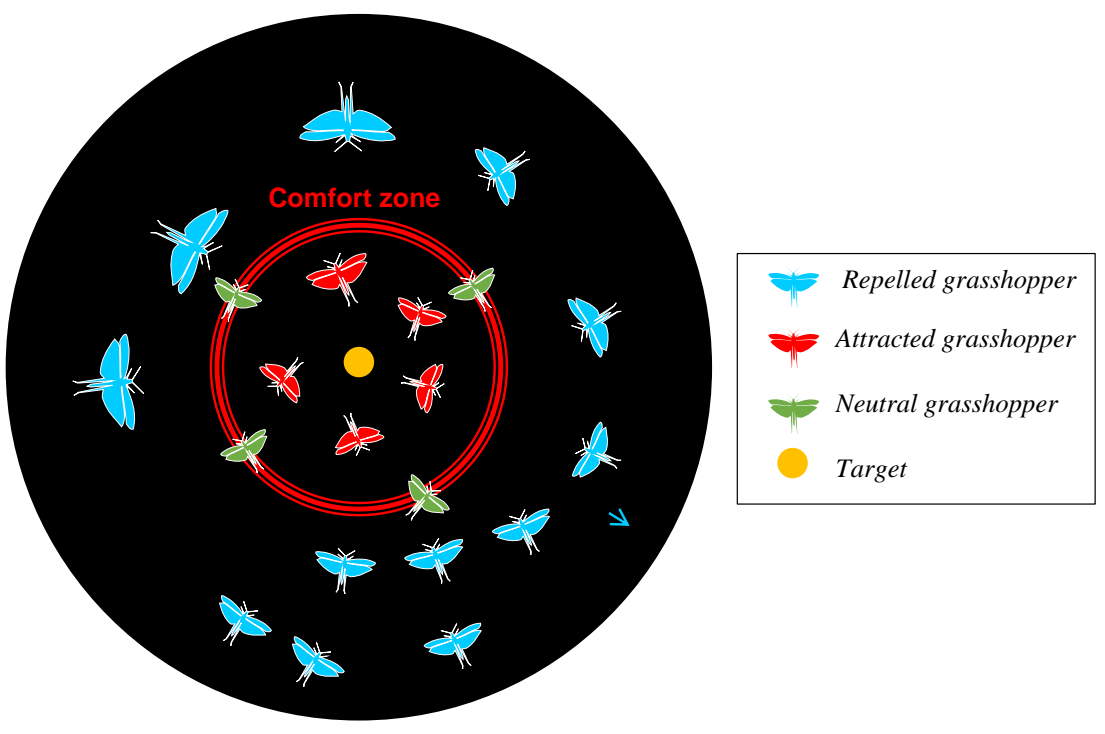

Fig. 1: Attraction, repulsion, and comfort zone in GOA

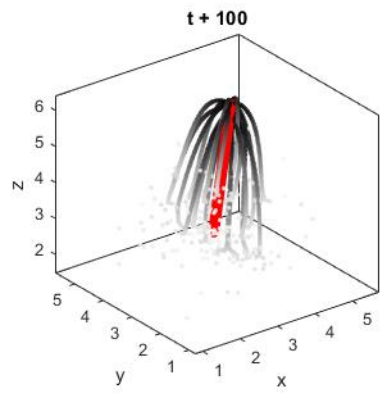

(a)

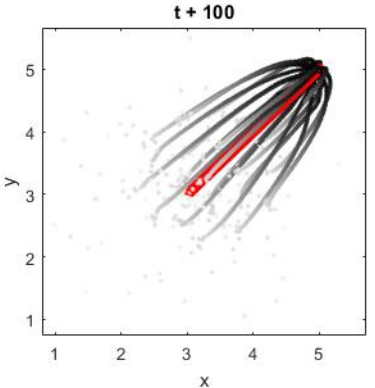

(b)

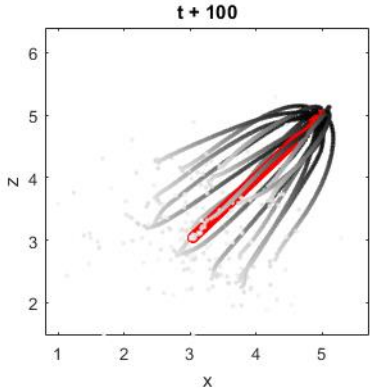

(c)

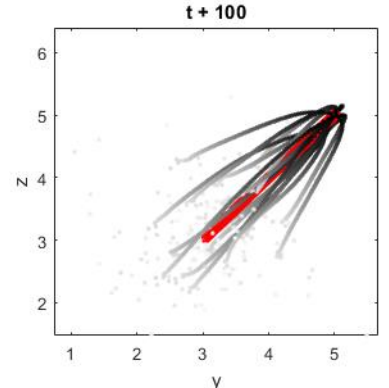

(d)

Fig. 2: Trajectory of swarm (a) in $3 \mathrm{D}$ space (b) $\mathrm{x}-\mathrm{y}$ view (c) $\mathrm{x}-\mathrm{z}$ view (d) $\mathrm{y}-\mathrm{z}$ view

\section{Support Vector Machines}

Support Vector Machine (SVM) is a non-linear binary classifier that was developed by Vladimir Vapnik [10, 42,43]. The are an extension of the Support Vector Classifier (SVC) to accommodate the non-linear boundaries between classes, which in turn is an extension of the Maximal Margin Classifier (MMC) that is limited to simple linearly-separable data [7]. SVMs are used in supervised learning and work for both classification and regression.

Formally, an SVM constructs linear separating hyperplanes in high-dimensional vector spaces in which each data point can be viewed as $\left(x_{i}, y_{i}\right)$ pair, where $x_{i}$ is the feature vector $\left(x_{i 1}, x_{i 2}, \ldots, x_{i p}\right) ; p$ is the number of features, $i=1, \ldots, n$, where $n$ is the number of training observations, and $y_{i}$ is the class label; usually $\{-1,1\}[17]$. If the feature space is well separated, several hyperplanes will exist that separate the data points belonging to the two labelled classes into separated regions in the space. The best hyperplane is defined as the one that maximizes the distance to the nearest training data points; this distance is called the margin. The observations that are far from the hyperplane by a distance equal to the margin are called support vectors $(\mathrm{SV})$. These vectors are located in the feature space. The hyperplane location depends directly and only on these observations SV, such that if any of these observations change their locations, the location of the hyperplane will also be changed accordingly [17]. Fig. 3 shows the illustration of the idea of the optimal hyperplane and support vectors in SVM.

The linear separating hyperplanes will lead to optimal classification. However, in most of the cases the data points that belong to different classes are not clearly separated, and hence, linear classification will lead to substantial misclassification. One way that SVMs can handle such frequent cases is the ability to map the orig- 
inal feature space (most likely non-linear) to a higherdimensional space $\varphi$, in which the data points that belong to different classes have clearer separation boundaries (become linearly separable). The high-dimensional space results from non-linear enlarging of the original space using kernel functions. Kernel functions have several forms such as:

- Linear kernels with the following form:

$$
k\left(x_{i}, x_{i}^{\prime}\right)=\sum_{j=1}^{p} x_{i j} x_{i j}^{\prime}
$$

- Polynomial kernels with the following form:

$$
k\left(x_{i}, x_{i}^{\prime}\right)=\left(1+\sum_{j=1}^{p} x_{i j} x_{i j}^{\prime}\right)^{d},
$$

- RBF kernels with the following form:

$$
k\left(x_{i}, x_{i}^{\prime}\right)=\exp \left(-\gamma \sum_{j=1}^{p}\left(x_{i j}-x_{i j}^{\prime}\right)^{2}\right),
$$

Where $k($.$) is the kernel function and its value is the$ inner product of the two observation vectors $x_{i}$ and $x_{i}^{\prime}$. In the transformed feature space $\varphi$ the inner product of two vectors can be represented as $\varphi\left(x_{i}\right) \cdot \varphi\left(x_{i}^{\prime}\right)$. A $\mathrm{SVM}$ is a support vector classifier with non-linear kernel function.

A major determinant of the performance of the SVM is the selection of its kernel function and their parameters. In this paper, we used the GOA algorithm for optimising these parameters.

\section{GOA for optimising SVM and feature selection}

In this section, we describe the methodology followed to use GOA for feature selection and optimising the parameters of SVM simultaneously. The methodology adopted is based on the wrapper based approach for feature selection. Wrapper is a type of feature selection method, which evaluates the selected subset of features based on their predictive measure. The main advantage of this approach is that it incorporates a learning algorithm to evaluate the searched subset of features. Wrapper based feature selection consists of three main components: a search method, an inductive algorithm, and an evaluation measurement [19]. In our implementation, GOA is used as a search algorithm for finding a good subset of features, SVM is used as an inductive algorithm, while classification accuracy is utilized as an evaluation measure. Fig. 4 shows the high level of the wrapper based approach that is used for feature selection based on GOA and SVM.

In order to apply any metaheuristic algorithm for optimising a problem, there are two formulation issues that should be addressed. The first is how the individual (i.e. solution) is represented, while the second is the selection of a fitness function used for evaluating the quality of the generated solutions. In our proposed GOA-SVM, we address both points as follows.

- Individual/Population representation: To address this point we must decide which decision variables to represent in the individual. In our case, these variables are the SVM parameters and the input features. Therefore, in our implementation, a single solution is represented as a one-dimensional vector of real numbers as shown in equation 5 . The first two elements of the vector are the candidate hyperparameters of the SVM, which are the cost $C$ and the kernel parameter $\gamma$. Other elements of the vector represent a sequence of flags, where each flag corresponds to a feature. That is, if the value of the flag is larger or equals to 0.5 then the feature is selected, otherwise the feature is excluded.

$I_{i}=\left[\begin{array}{lllll}C \gamma & F_{1} & F_{2} & \ldots & F_{n}\end{array}\right]$

- Fitness evaluation: The quality of every generated individual is assessed using a predefined measurement. For this purpose, a fitness function should be defined. In the proposed model, the fitness function relies on the average classification accuracy of the SVM models trained using the parameters and features indicated by the individual. This fitness function can be formulated as shown in equation 6 , and will be used to evaluate the quality of individual $I_{i}^{t}$ at iteration $t$, where $c\left(x_{j}\right)$ is the classification result of the $j$ th instance in the testing data set, $y_{j}$ is the actual class label of the $j$ th instance, $\delta$ is the relation between $c\left(x_{j}\right)$ and $y_{j}$ that is if $c\left(x_{j}\right)=y_{j}$ then $\delta=1$ otherwise $\delta=0 . N$ is the number of instances in the testing data set. Note that the fitness evaluation is measured based on $\mathrm{K}$-folds cross validation where $K$ denotes the number of folds. The latter step is important to avoid the problem of overfitting and consequently to obtain more robust results. This cross-validation will be referred to as an internal cross-validation.

$\operatorname{fitness}\left(I_{i}^{t}\right)=\frac{1}{K} \sum_{k=1}^{K} \frac{1}{N} \sum_{j=1}^{N} \delta\left(c\left(x_{j}\right), y_{j}\right)$

After deciding on these two design issues, the processes of running the GOA-SVM model can be described as follows:

1. Initialization: The GOA-SVM model starts with an initial random population of candidate solutions where 


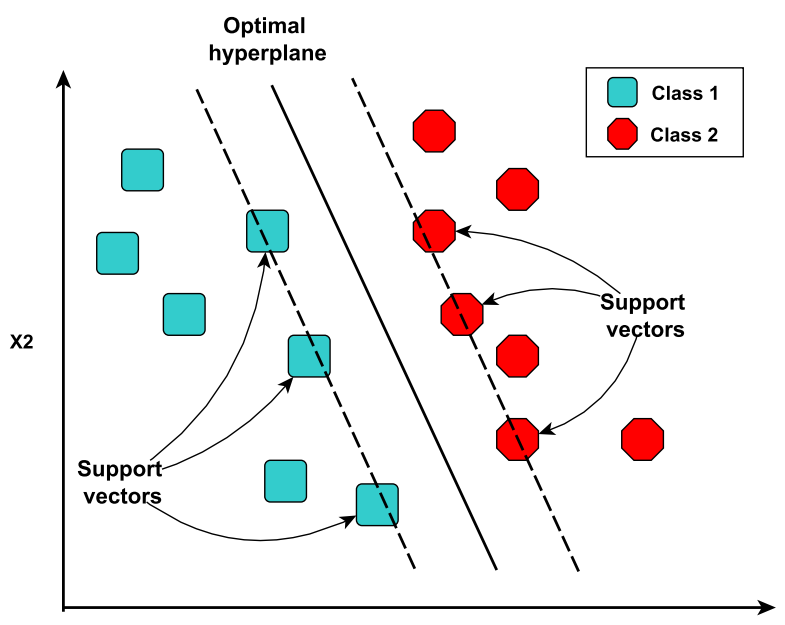

$\mathrm{X} 1$

Fig. 3: Illustration of the idea of the optimal hyperplane and support vectors in SVM

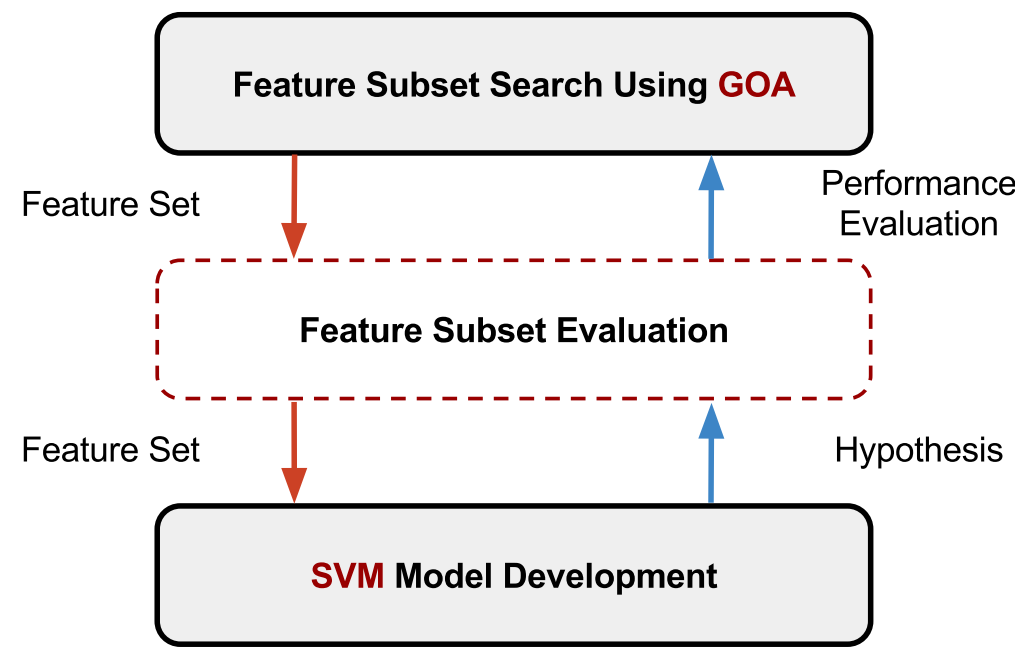

Fig. 4: Wrapper based approach for feature selection based on GOA and SVM

each solution consists of the $C$ and $\gamma$ parameters and a number of flags corresponding to the features in the data set as was shown in equation 5 .

2. Update and reproduction: reproduction operators of the GOA are applied in this step on all individuals to form a new population of candidate solutions.

3. Fitness evaluation: the quality of every generated individual is assessed using the fitness function, which was described previously in equation 6 .

4. Termination: the search process for the best solution ends when the maximum number of iterations is reached. Then, the GOA-SVM model reports the best SVM parameters along with the subset of fea- tures that was found to have the highest classification accuracy.

5. Testing: For verification, the final step is to test the developed SVM model with the selected parameters and features based on an unseen and new part of the original data set.

The overall process of the proposed GOA-SVM model can be summarized as depicted in the schematic diagram shown in Fig. 5. The diagram shows that the proposed approach starts by specifying the input data set. After that, some of the preprocessing operations are applied such as data normalization, and missing value handling. After this step, the training and testing parts 
are performed based on a cross-validation methodology. The training part is then used in the SVM parameters optimisation and the feature subset selection. After that, the optimisation process is started using the GOA algorithm to find the best SVM parameters and the most relevant feature subset of the SVM model. After this iterative process, the final SVM model is used to evaluate the testing data using the classification accuracy evaluation measure.

\section{Experiments and Results}

To evaluate and benchmark the proposed GOA-SVM approach, 18 binary and multi-class data sets were obtained from the University of California at Irvine (UCI) Machine Learning Repository ${ }^{1}$ [21]. The description of the data sets in terms of number of instances, number of features, and number of classes is given in Table 1. As can be seen, the data sets are varied in their size, and number of dimensions, in order to test the proposed approach on different scales of problem complexity. Moreover, most of the data sets (14 out of 18) are considered high-dimensional data sets, as they have more than 10 features/dimensions $[5,18]$. It is worth mentioning that many of these data sets have been used in the literature for testing SVM based classifiers.

To give an equal weight for all features, it is important to convert them all to the same scale. Therefore, all data sets are normalized before the training process. In this work we apply the Min-Max normalization, which can be defined as given in Equation 7 , where $F_{n e w}$ is the newly transformed feature, $\min _{F}$ and $\max _{F}$ are the minimum and maximum values of the targeted feature $F$. Using this Equation, all features are scaled to the interval $[0,1]$.

$F_{\text {new }}=\frac{F_{i}-\min _{F}}{\max _{F}-\min _{F}}$

As a training/testing methodology, we apply a 10folds cross validation, where each data set is divided into 10 equal parts. Nine parts are used for training and one for testing. Then, another nine parts are used for training and the 10th part is used for testing and so on. This processes is repeated 10 times. Finally, the average of the classification accuracy on testing parts is reported.

The GOA-SVM approach is compared to MVO, GA, PSO, GWO, FF, BA, and CS approaches using the 18 data sets in order to verify its performance. Furthermore, the comparison with the gird search method is

\footnotetext{
${ }^{1}$ http://archive.ics.uci.edu/ml/
}

discussed. To make the comparisons fair, the experiments are repeated for 10 times/runs to minimize random effects. Each run is set to 100 iterations as stopping criteria. Furthermore, all settings and parameters that are used in the experiments for all algorithms are presented in Table 2.

The implementation of the proposed model is developed on Matlab R2015a environment. Foe SVM, we used Libsvm [9]. The experimental evaluation was performed on a PC with an Intel Core i5-6400, 2.70 GHz, 8 GB RAM, and Windows 7.

Classification rate or accuracy is the main evaluation measure that is used to assess the performance of the GOA-SVM as well as the other algorithms. Accuracy measures the ratio of the correctly classified instances to the actual classes. For each experiment, Average (Avg), and Standard Deviation (Std) of the classification results are reported. In addition, the Wilcoxons test is used to check the statistical significance of the difference of the given classification results. In this paper, a $5 \%$ significance level is used in the Wilcoxons test to threshold the calculated $\mathrm{P}$-values.

In this paper, we preformed three different experiments, the first experiment is applied on the whole set of features for every data set (without feature selection), the second experiment is applied on a reduced set of features for every data set (with feature selection), and in the last experiment, the GOA-SVM is compared with 6 other algorithms (Grid search, MVO, GA, PSO, and GSA), for which the results were obtained directly from previous studies.

\subsection{Experiment I: results without applying feature selection}

In this experiment, we apply the GOA and other algorithms on 18 data sets and employing the complete set of features for each data set. The accuracy results of the GOA and other algorithms are presented in Table 3. The average accuracy values show that GOA outperforms the other metaheuristics and obtains the best performance on 11 data sets out of 18 data sets $(60 \%$ of the data sets). For the other 7 data sets, the average accuracy results of the GOA are very competitive. For example, the performance of the GOA on the Liver data set was better than three of other algorithms (MVO, GWO, and BA). Moreover, the proposed approach obtains the highest classification accuracies on high-dimensional data sets such as Sonar, Spectft, Libras, and Ionosphere. These results support the capability of the proposed approach to handle highdimensional data sets. In addition, GOA shows smaller 
Table 1: List of data sets

\begin{tabular}{lccc}
\hline data set & Features & Instances & Classes \\
\hline Breast Cancer & 10 & 683 & 2 \\
Wine & 13 & 178 & 3 \\
Heart & 13 & 270 & 2 \\
Parkinsons & 22 & 195 & 2 \\
Sonar & 60 & 208 & 2 \\
Vowel & 10 & 528 & 11 \\
Spectft & 44 & 276 & 2 \\
Ionosphere & 34 & 351 & 2 \\
German & 24 & 1000 & 2 \\
Vehicle & 18 & 846 & 4 \\
Australian & 15 & 653 & 2 \\
Diabetes & 8 & 768 & 2 \\
Liver & 6 & 345 & 2 \\
Glass & 9 & 214 & 6 \\
Arrhythmia & 297 & 452 & 16 \\
Cleveland & 13 & 303 & 4 \\
Libras & 90 & 360 & 15 \\
Teaching Assistant Evaluation (TAE) & 5 & 151 & 3 \\
\hline
\end{tabular}

Table 2: Initial parameters of the metaheuristic algorithms

\begin{tabular}{|c|c|c|}
\hline Algorithm & Parameter & Value \\
\hline \multirow[t]{4}{*}{ GOA [34] } & cMin & 0.00001 \\
\hline & cMax & 1 \\
\hline & Number of search agents & 50 \\
\hline & Number of iterations & 100 \\
\hline \multirow[t]{5}{*}{ GA $[14]$} & Crossover ratio & 0.9 \\
\hline & Mutation ratio & 0.1 \\
\hline & Selection mechanism & Roulette wheel \\
\hline & Population size & 50 \\
\hline & Generations & 100 \\
\hline \multirow[t]{4}{*}{$\mathrm{PSO}[47,51]$} & Acceleration constants & {$[2.1,2.1]$} \\
\hline & Inertia $\mathrm{w}$ & {$[0.9,0.6]$} \\
\hline & Number of particles & 50 \\
\hline & Generations & 100 \\
\hline \multirow[t]{4}{*}{ MVO [25] } & Min wormhole existence ratio & 0.2 \\
\hline & Max wormhole existence ratio & 1 \\
\hline & Universes & 50 \\
\hline & Iterations & 100 \\
\hline \multirow[t]{4}{*}{$\mathrm{BA}[53]$} & Loudness & 0.5 \\
\hline & Pulse rate & 0.5 \\
\hline & Frequency minimum & 0 \\
\hline & Frequency maximum & 2 \\
\hline \multirow[t]{3}{*}{$\mathrm{FF}[52]$} & Alpha & 0.5 \\
\hline & Beta & 0.2 \\
\hline & Gamma & 1 \\
\hline \multirow[t]{3}{*}{ Cuckoo Search $[50]$} & Discovery rate $P \alpha$ & 0.25 \\
\hline & Number of nests & 50 \\
\hline & Number of generations & 100 \\
\hline GWO [26] & $\alpha$ & $\operatorname{Min}=0$, and $\operatorname{Max}=2$ \\
\hline
\end{tabular}




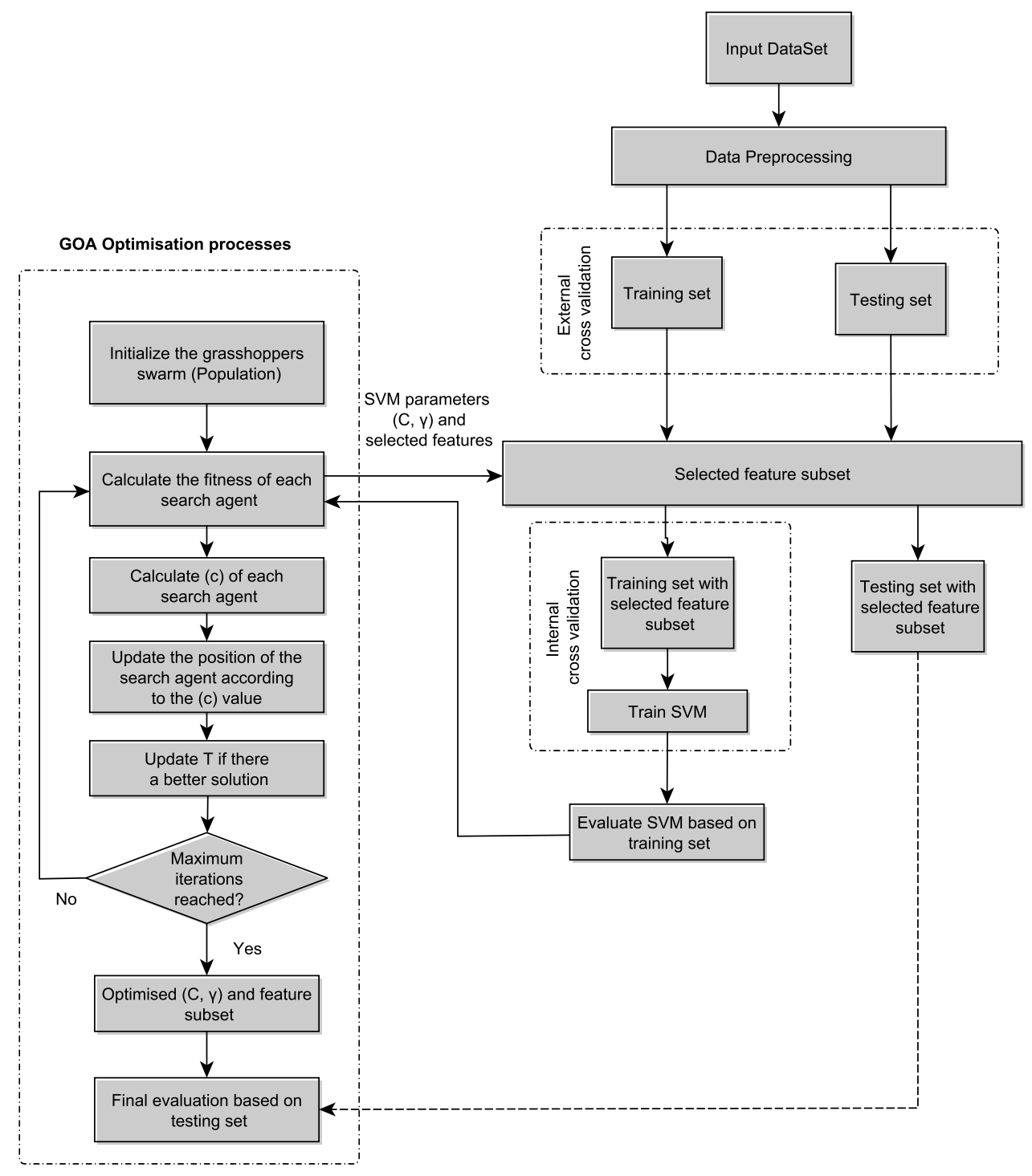

Fig. 5: Schematic diagram of the proposed GOA-SVM model.

standard deviation values for most of the data sets, which supports the robustness of the algorithm.

Fig. 3 presents the box-plot charts of the accuracy for all used data sets. The box-plots are shown for the 10 values of classification accuracy that are given by each algorithm at the end of the SVM training. In this plot, the box represents the interquartile range (IQR), the whiskers represent the maximum accuracy values, the bar in the box represents the median accuracy values, and outliers of the accuracy values are expressed as small circles. The box-plots indicate the improved performance of GOA for optimising the SVM.

To assess the overall performance of GOA and other algorithms, and to confirm the significance and robustness of the results, we apply the Wilcoxons statistical test with $5 \%$ significance level on the obtained average accuracy results. Table 4 lists Wilcoxons test P-values between the GOA and other approaches. The P-values less than 0.05 indicate that the GOA results have statistically significant differences compared with other approaches. The P-values that are greater than 0.05 (underlined) are not significant compared with other approaches. According to this table, the GOA results are statistically significant in most of the data sets.

As an overall summary for all data sets, Table 5 shows summarised values which represent the number of data sets each algorithm Won/Tied/Lost on accuracy, and the associated p-values. It appears that the GOA algorithm is superior on 11 data sets out of 18 in terms of accuracy. Moreover, in terms of significance, the results show that the GOA is better on $4,5,7,8$, 6,8 , and 4 data sets compared with MVO, GA, PSO, 


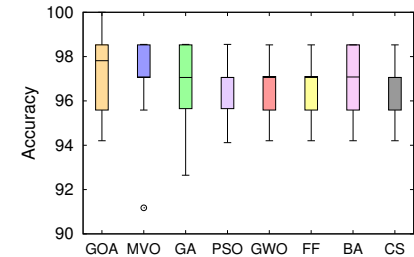

(a) Breast

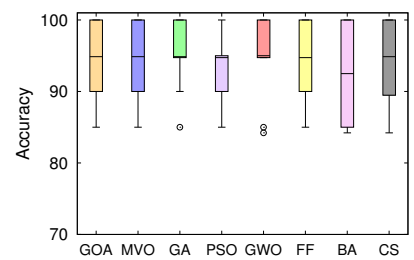

(d) Parkinsons

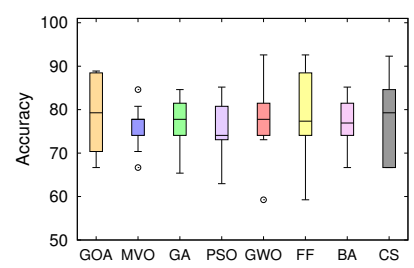

(g) Spectft

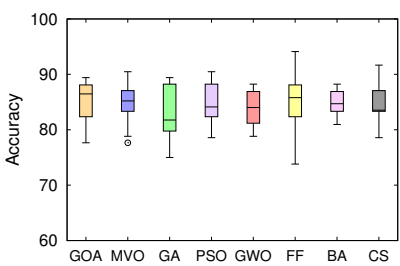

(j) Vehicle

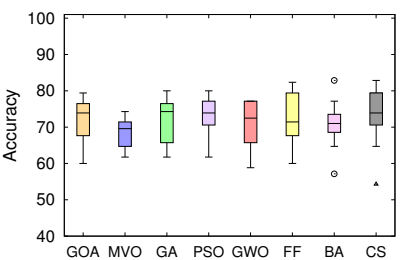

(m) Liver

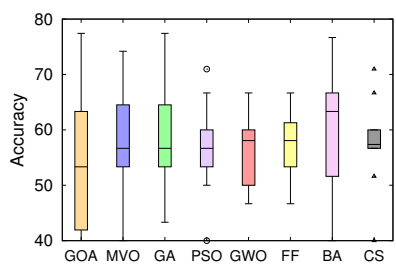

(p) Cleveland

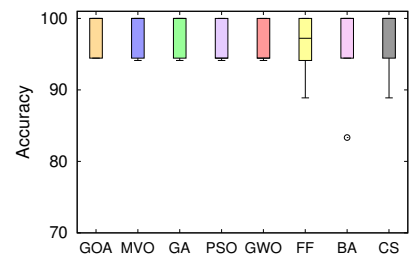

(b) Wine

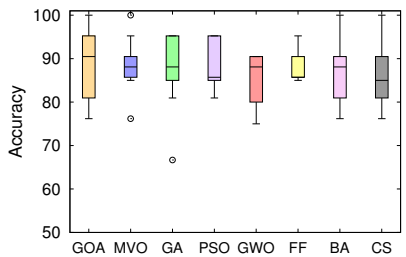

(e) Sonar

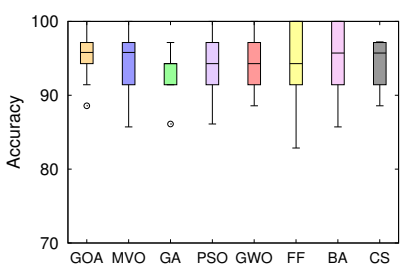

(h) Ionosphere

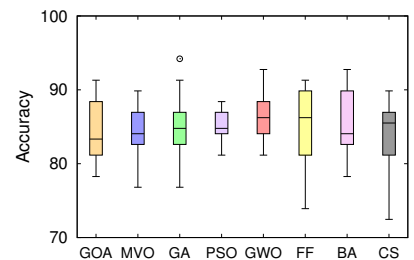

(k) Australian

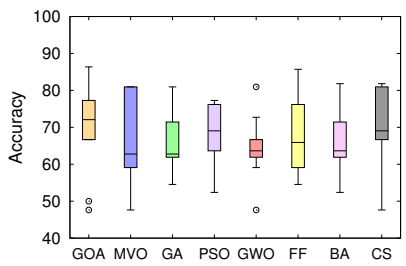

(n) Glass

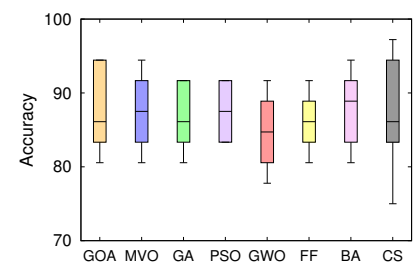

(q) Libras

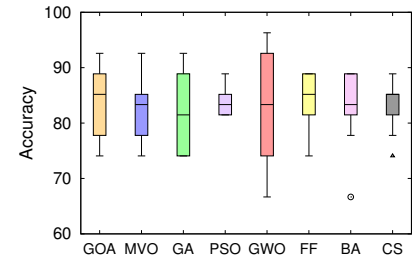

(c) Heart

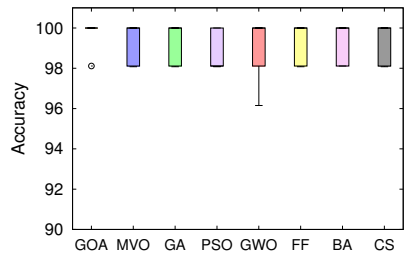

(f) Vowel

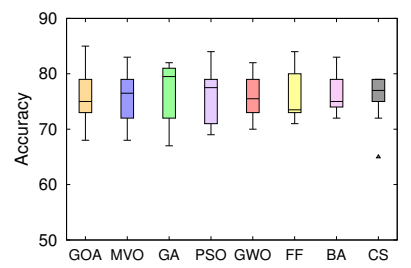

(i) German

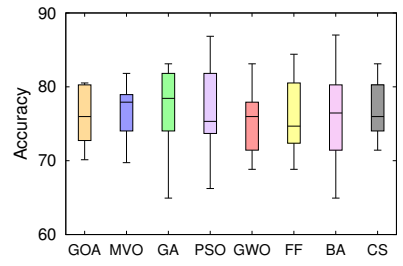

(l) Diabetes

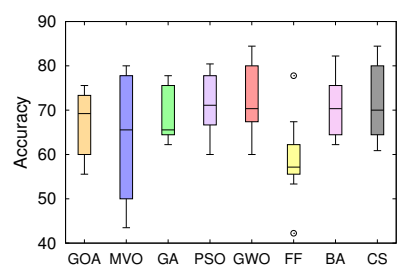

(o) Arrhythmia

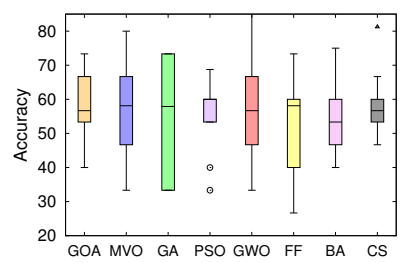

(r) Tae

Fig. 6: Box-plot charts for GOA and other algorithms based on 18 data sets (Without Features selection) 
Table 3: Classification accuracy and standard deviation results for all data sets without applying feature selection.

\begin{tabular}{|c|c|c|c|c|}
\hline Algorithm & $\mathrm{GOA}$ & MVO & GA & $\mathrm{PSO}$ \\
\hline data set & $\operatorname{Avg} \pm \operatorname{Std}$ & $\operatorname{Avg} \pm \operatorname{Std}$ & $\operatorname{Avg} \pm$ Std & $\operatorname{Avg} \pm \operatorname{Std}$ \\
\hline Breast Cancer & $97.23 \pm 1.88$ & $96.92 \pm 2.13$ & $96.78 \pm 1.94$ & $96.63 \pm 1.15$ \\
\hline Wine & $97.77 \pm 2.72$ & $97.75 \pm 2.76$ & $97.71 \pm 2.80$ & $96.60 \pm 2.78$ \\
\hline Heart & $82.22 \pm 6.24$ & $82.59 \pm 5.51$ & $82.59 \pm 7.23$ & $83.70 \pm 2.46$ \\
\hline Parkinsons & $\mathbf{9 4 . 9 5} \pm \mathbf{5 . 0 0}$ & $94.89 \pm 5.05$ & $94.92 \pm 4.47$ & $93.37 \pm 4.52$ \\
\hline Sonar & $88.55 \pm 8.01$ & $88.50 \pm 6.14$ & $87.52 \pm 8.57$ & $87.98 \pm 5.34$ \\
\hline Vowel & $99.81 \pm 0.57$ & $99.43 \pm 0.88$ & $99.43 \pm 0.88$ & $98.86 \pm 0.93$ \\
\hline spectft & $78.73 \pm 7.82$ & $76.45 \pm 4.81$ & $76.79 \pm 6.45$ & $75.31 \pm 5.91$ \\
\hline Ionosphere & $95.16 \pm 3.14$ & $94.59 \pm 3.93$ & $93.18 \pm 3.09$ & $94.04 \pm 4.05$ \\
\hline German & $75.80 \pm 5.31$ & $75.70 \pm 4.45$ & $76.80 \pm 5.27$ & $76.30 \pm 4.94$ \\
\hline Vehicle & $85.35 \pm 3.48$ & $84.76 \pm 3.93$ & $82.96 \pm 5.02$ & $85.10 \pm 3.63$ \\
\hline Australian & $84.93 \pm 4.06$ & $84.35 \pm 3.54$ & $85.07 \pm 4.72$ & $84.78 \pm 2.27$ \\
\hline diabetes & $76.05 \pm 3.72$ & $76.17 \pm 3.81$ & $76.44 \pm 6.43$ & $76.83 \pm 6.48$ \\
\hline liver & $71.60 \pm 6.17$ & $68.94 \pm 4.02$ & $72.13 \pm 6.28$ & $73.32 \pm 5.24$ \\
\hline glass & $70.06 \pm 11.84$ & $65.97 \pm 11.10$ & $65.48 \pm 8.34$ & $67.77 \pm 8.37$ \\
\hline Arrhythmia & $65.24 \pm 10.91$ & $64.24 \pm 13.26$ & $68.78 \pm 5.95$ & $71.21 \pm 6.67$ \\
\hline Cleveland & $55.08 \pm 12.07$ & $58.01 \pm 8.93$ & $59.01 \pm 8.61$ & $57.72 \pm 7.81$ \\
\hline Libras & $87.78 \pm 5.30$ & $87.22 \pm 5.00$ & $87.22 \pm 3.97$ & $87.50 \pm 3.34$ \\
\hline TAE & $58.25 \pm 10.42$ & $56.29 \pm 13.03$ & $54.92 \pm 16.37$ & $55.54 \pm 10.59$ \\
\hline Algorithm & GWO & $\mathrm{FF}$ & $\mathrm{BA}$ & CS \\
\hline data set & $\operatorname{Avg} \pm$ Std & $\operatorname{Avg} \pm$ Std & $\operatorname{Avg} \pm$ Std & $\operatorname{Avg} \pm$ Std \\
\hline Breast Cancer & $96.64 \pm 1.30$ & $96.64 \pm 1.30$ & $96.93 \pm 1.37$ & $96.49 \pm 1.47$ \\
\hline Wine & $96.63 \pm 2.75$ & $96.08 \pm 4.35$ & $97.22 \pm 5.12$ & $97.75 \pm 3.72$ \\
\hline Heart & $82.96 \pm 9.54$ & $84.44 \pm 4.91$ & $82.96 \pm 6.67$ & $83.33 \pm 4.46$ \\
\hline Parkinsons & $94.89 \pm 5.62$ & $93.89 \pm 4.93$ & $92.79 \pm 6.63$ & $93.82 \pm 5.55$ \\
\hline Sonar & $85.50 \pm 5.93$ & $88.45 \pm 3.85$ & $88.02 \pm 7.48$ & $85.10 \pm 6.88$ \\
\hline Vowel & $99.05 \pm 1.28$ & $99.24 \pm 0.93$ & $99.43 \pm 0.86$ & $99.24 \pm 0.93$ \\
\hline spectft & $77.54 \pm 8.12$ & $77.19 \pm 10.75$ & $77.54 \pm 5.46$ & $77.25 \pm 8.71$ \\
\hline Ionosphere & $94.30 \pm 3.13$ & $94.57 \pm 5.34$ & $94.86 \pm 4.75$ & $94.29 \pm 3.14$ \\
\hline German & $76.10 \pm 3.78$ & $76.90 \pm 4.20$ & $76.30 \pm 3.49$ & $75.80 \pm 4.19$ \\
\hline Vehicle & $83.93 \pm 3.02$ & $84.87 \pm 5.45$ & $84.75 \pm 2.19$ & $84.64 \pm 3.39$ \\
\hline Australian & $86.38 \pm 3.50$ & $85.36 \pm 5.36$ & $85.07 \pm 4.25$ & $84.06 \pm 4.85$ \\
\hline diabetes & $75.91 \pm 4.55$ & $75.77 \pm 4.83$ & $76.31 \pm 6.50$ & $76.70 \pm 3.54$ \\
\hline liver & $71.27 \pm 6.04$ & $71.91 \pm 7.37$ & $71.00 \pm 6.55$ & $72.48 \pm 7.79$ \\
\hline glass & $64.48 \pm 8.23$ & $67.88 \pm 9.97$ & $67.27 \pm 8.67$ & $69.09 \pm 10.83$ \\
\hline Arrhythmia & $71.92 \pm 7.23$ & $59.06 \pm 8.88$ & $71.24 \pm 6.37$ & $71.72 \pm 7.97$ \\
\hline Cleveland & $56.44 \pm 6.17$ & $57.09 \pm 5.19$ & $59.78 \pm 11.28$ & $57.73 \pm 7.88$ \\
\hline Libras & $84.72 \pm 4.17$ & $86.39 \pm 3.61$ & $87.22 \pm 7.26$ & $86.67 \pm 6.89$ \\
\hline TAE & $57.71 \pm 14.57$ & $52.96 \pm 15.08$ & $55.50 \pm 11.45$ & $58.13 \pm 9.75$ \\
\hline
\end{tabular}

GWO, FF, BA, and CS, respectively. In addition, the Friedman test was used to rank the different algorithms applied to 18 data sets. The results of the Friedman test show that the GOA obtains the best rank (Lower is better). This confirms the ability of the GOA algorithm to optimise the parameters of the SVM classifier.
6.2 Experiment II: results with applying feature selection

In this experiment, we apply the GOA and other algorithms on 18 data sets, when extending the GOA for simultaneously optimising the SVM parameters and performing feature selection. Table 6 shows the results when applying GOA with feature selection compared with GOA without feature selection. The results show 
Table 4: P-values of the Wilcoxon test of GOA vs other algorithms before applying features selection ( $\mathrm{p} \geq 0.05$ are underlined).

\begin{tabular}{|c|c|c|c|c|c|c|c|}
\hline data set/Algorithm & MVO & GA & PSO & GWO & $\mathrm{FF}$ & $\mathrm{BA}$ & CS \\
\hline Breast Cancer & $9.02 \mathrm{E}-01$ & $1.67 \mathrm{E}-01$ & $7.72 \mathrm{E}-04$ & $2.74 \mathrm{E}-03$ & $2.74 \mathrm{E}-03$ & $7.85 \mathrm{E}-02$ & $2.75 \mathrm{E}-04$ \\
\hline Wine & $\underline{5.71 \mathrm{E}-01}$ & $2.64 \mathrm{E}-02$ & $1.45 \mathrm{E}-04$ & $5.72 \mathrm{E}-09$ & $5.40 \mathrm{E}-05$ & 7.74E-01 & $\underline{7.74 \mathrm{E}-01}$ \\
\hline Heart & $\underline{6.17 \mathrm{E}-01}$ & $\underline{8.05 \mathrm{E}-01}$ & $1.00 \mathrm{E}+00$ & 7.12E-01 & $1.01 \mathrm{E}-01$ & $\underline{5.33 \mathrm{E}-01}$ & $1.00 \mathrm{E}+00$ \\
\hline Parkinsons & $\underline{8.00 \mathrm{E}-01}$ & $\underline{9.00 \mathrm{E}-01}$ & $5.78 \mathrm{E}-03$ & $\underline{4.46 \mathrm{E}-01}$ & $\underline{7.78 \mathrm{E}-02}$ & $2.26 \mathrm{E}-02$ & $1.67 \mathrm{E}-01$ \\
\hline Sonar & $\underline{4.57 \mathrm{E}-01}$ & $\underline{6.21 \mathrm{E}-01}$ & $\underline{3.86 \mathrm{E}-01}$ & $1.69 \mathrm{E}-04$ & $\underline{2.62 \mathrm{E}-01}$ & $\underline{3.86 \mathrm{E}-01}$ & $5.39 \mathrm{E}-04$ \\
\hline Vowel & $1.16 \mathrm{E}-04$ & $2.37 \mathrm{E}-04$ & $9.00 \mathrm{E}-14$ & $1.40 \mathrm{E}-06$ & $6.44 \mathrm{E}-06$ & $2.86 \mathrm{E}-03$ & $6.44 \mathrm{E}-06$ \\
\hline Spectft & $1.89 \mathrm{E}-02$ & $1.10 \mathrm{E}-01$ & $3.11 \mathrm{E}-03$ & $\underline{5.39 \mathrm{E}-01}$ & $\underline{6.24 \mathrm{E}-01}$ & $\underline{2.19 \mathrm{E}-01}$ & $1.40 \mathrm{E}-01$ \\
\hline Ionosphere & $\underline{6.13 \mathrm{E}-01}$ & $8.67 \mathrm{E}-07$ & $3.21 \mathrm{E}-02$ & $1.74 \mathrm{E}-02$ & $7.09 \mathrm{E}-01$ & $\underline{9.01 \mathrm{E}-01}$ & $2.04 \mathrm{E}-01$ \\
\hline German & $\underline{6.25 \mathrm{E}-01}$ & $\underline{6.61 \mathrm{E}-02}$ & $\underline{3.27 \mathrm{E}-01}$ & $\underline{6.23 \mathrm{E}-01}$ & $\underline{3.26 \mathrm{E}-01}$ & $\underline{4.60 \mathrm{E}-01}$ & $\underline{6.41 \mathrm{E}-02}$ \\
\hline Vehicle & $\underline{2.20 \mathrm{E}-01}$ & $3.27 \mathrm{E}-03$ & $\underline{6.24 \mathrm{E}-01}$ & $1.45 \mathrm{E}-03$ & $\underline{4.63 \mathrm{E}-01}$ & $3.28 \mathrm{E}-03$ & $4.98 \mathrm{E}-02$ \\
\hline Australian & $\underline{8.06 \mathrm{E}-01}$ & $\underline{3.89 \mathrm{E}-01}$ & $\underline{4.59 \mathrm{E}-01}$ & $1.37 \mathrm{E}-03$ & $\underline{1.09 \mathrm{E}-01}$ & $\underline{2.67 \mathrm{E}-01}$ & $\underline{9.03 \mathrm{E}-01}$ \\
\hline Diabetes & $\underline{8.07 \mathrm{E}-01}$ & $4.99 \mathrm{E}-02$ & $2.70 \mathrm{E}-01$ & $6.25 \mathrm{E}-01$ & $\underline{6.24 \mathrm{E}-01}$ & 9.03E-01 & 3.27E-01 \\
\hline Liver & $2.86 \mathrm{E}-05$ & 2.19E-01 & $\underline{8.58 \mathrm{E}-02}$ & $6.24 \mathrm{E}-01$ & $\underline{3.91 \mathrm{E}-01}$ & $4.98 \mathrm{E}-02$ & $\underline{3.25 \mathrm{E}-01}$ \\
\hline Glass & $6.87 \mathrm{E}-03$ & $1.40 \mathrm{E}-04$ & $2.70 \mathrm{E}-02$ & $4.83 \mathrm{E}-07$ & $2.74 \mathrm{E}-02$ & $2.10 \mathrm{E}-03$ & $\underline{3.25 \mathrm{E}-01}$ \\
\hline Arrhythmia & $\underline{8.07 \mathrm{E}-01}$ & $\underline{1.11 \mathrm{E}-01}$ & $2.34 \mathrm{E}-04$ & $9.37 \mathrm{E}-04$ & $2.66 \mathrm{E}-07$ & $9.37 \mathrm{E}-04$ & $1.45 \mathrm{E}-03$ \\
\hline Cleveland & $\underline{6.59 \mathrm{E}-02}$ & $1.41 \mathrm{E}-02$ & $1.41 \mathrm{E}-01$ & $\underline{6.24 \mathrm{E}-01}$ & $3.26 \mathrm{E}-01$ & $2.93 \mathrm{E}-03$ & $1.77 \mathrm{E}-01$ \\
\hline Libras & $\underline{3.81 \mathrm{E}-01}$ & $5.32 \mathrm{E}-01$ & $9.01 \mathrm{E}-01$ & $1.12 \mathrm{E}-04$ & $1.03 \mathrm{E}-01$ & $1.00 \mathrm{E}+00$ & 7.09E-01 \\
\hline TAE & $\underline{3.89 \mathrm{E}-01}$ & $\underline{6.53 \mathrm{E}-02}$ & $\underline{2.14 \mathrm{E}-01}$ & $\underline{1.41 \mathrm{E}-01}$ & $1.39 \mathrm{E}-02$ & 8.39E-02 & $\underline{1.76 \mathrm{E}-01}$ \\
\hline
\end{tabular}

Table 5: Summary statistical results on a variety of measures (without feature selection). The table values represent the number of data sets each algorithm won/losses/ties on a variety of measures along with their rank using F-test.

\begin{tabular}{|c|c|c|c|c|c|c|c|c|c|c|c|c|c|c|c|c|c|c|c|c|c|c|c|c|}
\hline \multirow[t]{2}{*}{ Algorithm } & \multicolumn{3}{|c|}{ GOA } & \multicolumn{3}{|c|}{ MVO } & \multicolumn{3}{|c|}{ GA } & \multicolumn{3}{|c|}{ PSO } & \multicolumn{3}{|c|}{ GWO } & \multicolumn{3}{|c|}{ FF } & \multicolumn{3}{|c|}{ BA } & \multicolumn{3}{|c|}{$\mathrm{CS}$} \\
\hline & W & $\mathrm{T}$ & $\mathrm{L}$ & $\mathrm{W}$ & $\mathrm{T}$ & $\mathrm{L}$ & W & $\mathrm{T}$ & $\mathrm{L}$ & W & $\mathrm{T}$ & $\mathrm{L}$ & W & $\mathrm{T}$ & $\mathrm{L}$ & W & $\mathrm{T}$ & $\mathrm{L}$ & W & $\mathrm{T}$ & $\mathrm{L}$ & W & $\mathrm{T}$ & $\mathrm{L}$ \\
\hline Accuracy & 11 & 0 & 7 & 0 & 0 & 18 & 0 & 0 & 18 & 2 & 0 & 16 & 2 & 0 & 16 & 2 & 0 & 16 & 1 & 0 & 17 & 0 & 0 & 18 \\
\hline $\mathrm{P}$-value & $\mathrm{N} / \mathrm{A}$ & $\mathrm{N} / \mathrm{A}$ & $\mathrm{N} / \mathrm{A}$ & 0 & 14 & 4 & 2 & 11 & 5 & 1 & 10 & 7 & 2 & 8 & 8 & 0 & 12 & 6 & 1 & 9 & 8 & 1 & 13 & 4 \\
\hline Rank & & 3.08 & & & 4.81 & & & 4.67 & & & 4.69 & & & 5.22 & & & 4.83 & & & 4.06 & & & 4.64 & \\
\hline
\end{tabular}

that the effect of feature selection improves the results. As we can see in the table, the accuracy results are improved for 10 data sets with clear reduction of the original features ( $32 \%$ to $65 \%$ reduction rate). In addition, the results for the remaining 8 data sets with feature selection are very close to the results with all features. These results indicate that the proposed feature selection approach, used simultaneously with SVM parameter optimisation, is able to get better accuracy results with a considerable reduction in the number of original features.

For high-dimensional data sets such as Libras, Arrhythmia, Ionosphere, Spectft, and Sonar, their results show that the features are reduced to $52.22 \%, 53.09 \%$, $55.59 \%, 55.00 \%$ and $47.00 \%$, respectively. In addition, the accuracy rates for these data sets are higher than the results with all features. These results reveal that the proposed approach not only locates the optimal model parameters and reduces the number of features, but also can find the optimal and most relevant features.

Table 7 shows the comparison of GOA and other algorithms after applying the feature selection. It is noted that GOA outperforms the other algorithms in 12 data sets in terms of classification accuracy. Moreover, it shows competitive results in terms of minimizing the number of selected features.

Fig. 7 shows the box-plots of the accuracy results with feature selection for all of the data sets used. The box-plots confirm that the results of the GOA approach has higher median whilst maintaining smaller interquartile ranges in most cases. This proves that the GOA algorithm is better than others in terms of stability and robustness.

Table 8 shows the obtained $\mathrm{P}$-values from the Wilcoxons rank test between the GOA algorithm and other algorithms for all data sets after applying feature selection. The table shows that the accuracy results of the 
Table 6: Classification accuracy and standard deviation results of GOA for all data sets with and without applying feature selection.

\begin{tabular}{|c|c|c|c|c|c|}
\hline \multirow{2}{*}{$\begin{array}{l}\text { Algorithm } \\
\text { data set }\end{array}$} & \multicolumn{2}{|c|}{ Without feature selection } & \multicolumn{3}{|c|}{ With feature selection } \\
\hline & $\begin{array}{l}\text { Accuracy } \\
(\text { Avg } \pm \text { Std })\end{array}$ & $\begin{array}{c}\text { Original } \\
\text { No. of features }\end{array}$ & $\begin{array}{l}\text { Accuracy } \\
(\text { Avg士Std) }\end{array}$ & $\begin{array}{l}\text { No. of selected } \\
\text { features }(\operatorname{Avg} \pm \text { Std })\end{array}$ & $\begin{array}{l}\text { Reduction } \\
\text { rate }(\%)\end{array}$ \\
\hline Breast cancer & $\mathbf{9 7 . 2 3} \pm \mathbf{1 . 8 8}$ & 10 & $96.49 \pm 1.17$ & $6.70 \pm 1.19$ & 33.00 \\
\hline Wine & $97.77 \pm 2.72$ & 13 & $97.77 \pm 2.72$ & $7.70 \pm 1.35$ & 40.77 \\
\hline Heart & $82.22 \pm 6.24$ & 13 & $82.59 \pm 6.20$ & $7.10 \pm 1.22$ & 45.38 \\
\hline Parkinsons & $94.95 \pm 5.00$ & 22 & $95.97 \pm 4.37$ & $12.90 \pm 1.92$ & 41.36 \\
\hline Sonar & $88.55 \pm 8.01$ & 60 & $88.86 \pm 5.93$ & $31.80 \pm 3.54$ & 47.00 \\
\hline Vowel & $\mathbf{9 9 . 8 1} \pm 0.57$ & 10 & $99.05 \pm 1.27$ & $7.40 \pm 0.92$ & 26.00 \\
\hline Spectft & $78.73 \pm 7.82$ & 44 & $80.52 \pm 5.44$ & $19.80 \pm 3.16$ & 55.00 \\
\hline Ionosphere & $95.16 \pm 3.14$ & 34 & $93.44 \pm 2.88$ & $16.80 \pm 2.27$ & 50.59 \\
\hline German & $75.80 \pm 5.31$ & 24 & $73.4 \pm 4.45$ & $10.00 \pm 3.03$ & 58.33 \\
\hline Vehicle & $85.35 \pm 3.48$ & 18 & $82.50 \pm 2.12$ & $11.40 \pm 1.28$ & 36.67 \\
\hline Australian & $84.93 \pm 4.06$ & 15 & $85.07 \pm 5.27$ & $5.40 \pm 2.00$ & 64.00 \\
\hline Diabetes & $76.05 \pm 3.72$ & 8 & $74.35 \pm 5.15$ & $4.10 \pm 1.58$ & 48.75 \\
\hline liver & $71.60 \pm 6.17$ & 6 & $67.51 \pm 8.05$ & $4.20 \pm 1.47$ & 30.00 \\
\hline Glass & $70.06 \pm 11.84$ & 9 & $69.19 \pm 10.72$ & $5.20 \pm 1.17$ & 42.22 \\
\hline Arrhythmia & $65.24 \pm 10.91$ & 297 & $70.35 \pm 4.75$ & $142.30 \pm 4.69$ & 52.09 \\
\hline Cleveland & $55.08 \pm 12.07$ & 13 & $55.47 \pm 7.06$ & $6.90 \pm 2.12$ & 46.92 \\
\hline Libras & $87.78 \pm 5.30$ & 90 & $89.44 \pm 4.44$ & $43.00 \pm 4.31$ & 52.22 \\
\hline TAE & $58.25 \pm 10.42$ & 5 & $58.91 \pm 7.85$ & $3.40 \pm 1.02$ & 32.00 \\
\hline
\end{tabular}

GOA are significantly improved for most of the data sets.

As an overall summary for all data sets, Table 9 shows the number Wins, Ties, and Losses on accuracy, minimum number of features, and p-values measures for each algorithm. It appears that the GOA algorithm is superior on 12 data sets out of 18 in terms of accuracy. Moreover, in term of statistical significance, the results show that the GOA are better on $9,4,6,7,7,10$, and 7 data sets compared with MVO, GA, PSO, GWO, FF, $\mathrm{BA}$, and $\mathrm{CS}$, respectively. In addition, the Friedman test shows that the GOA obtains the best rank.

6.3 Experiment III: comparisons with metaheuristics in the literature

In this section, we compare the results of the optimised SVM using GOA with grid search, and four popular metaheuristics algorithms (MVO, GA, PSO, and GSA) that are obtained directly from the literature (twelve data sets used). The comparison is conducted based on the system architecture used in these works $[16,22,20]$. Table 10 shows more details about the approaches that are used for the comparisons.

Table 11 shows the results of the GOA and other well-regarded approaches. It can be seen that GOA outperforms the gird search method in all data sets with notable differences in the accuracy results. In addition, GOA outperforms the other metaheuristics algorithms and performs reasonably in eight data sets out of twelve. Moreover, the results of the GOA for the other four data sets are very close to the other algorithms.

Taken together, the results of this section showed that the GOA along with SVM can make a very effective memetic, wrapper-based algorithm to solve a wider range of classification problems. For one, the GOA algorithm addressed the high-dimensional nature of feature selection problems. This algorithm has been equipped with effective operators to avoid the local solutions of such problems and find a reasonable estimation of the global optimum in a reasonable time. For another, the SVM used the selected feature by the GOA algorithm to classify the data sets with an accurate classification rate. The outcome of using GOA and SVM to handle difficulties in two steps of classification proven to be very effective as per the results of this section.

As mentioned earlier, the No Free Lunch theorem states and has been logically proved that there is no general optimiser to solve all optimisation problems. However, some algorithms might be beneficial for a set of problems with or without modification or hybridization. The operator integrated in GOA make it suitable for selecting an optimal set of feature from a give large set of features. In fact, this algorithm benefits from a 


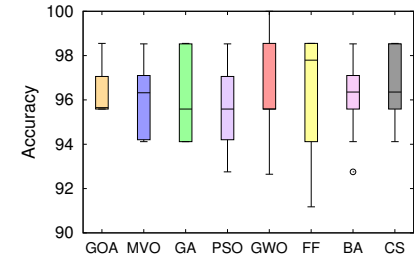

(a) breast

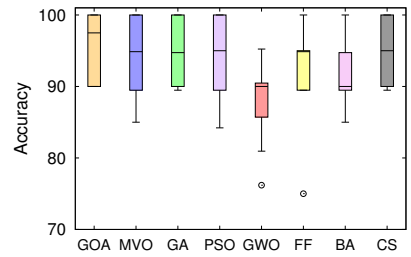

(d) Parkinsons

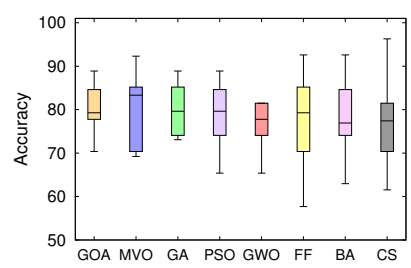

(g) Spectft

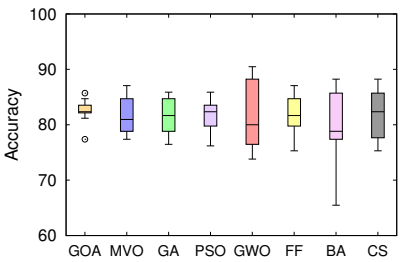

(j) Vehicle

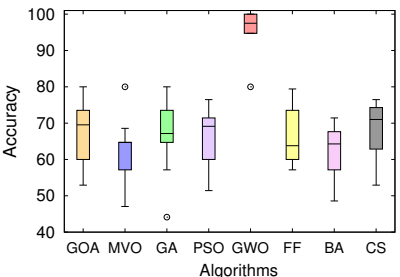

(m) Liver

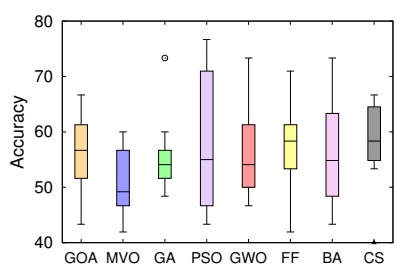

(p) Cleveland

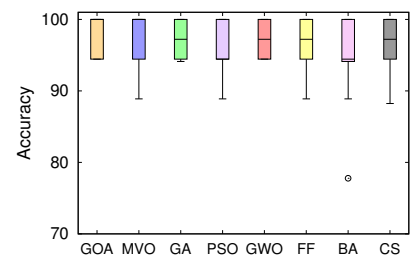

(b) wine

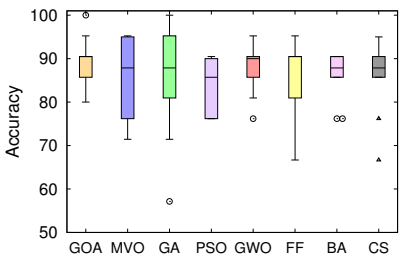

(e) Sonar

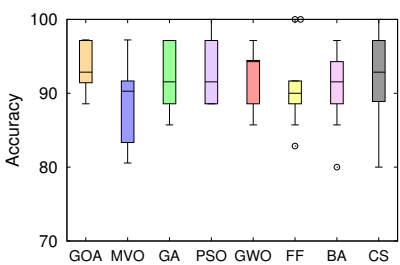

(h) Ionosphere

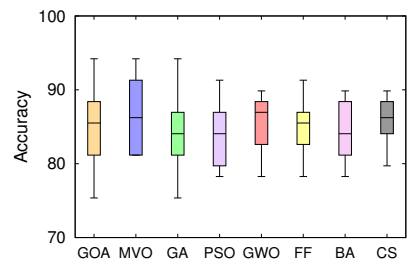

(k) Australian

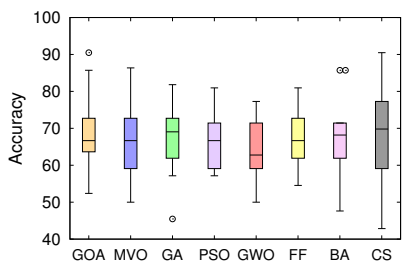

(n) Glass

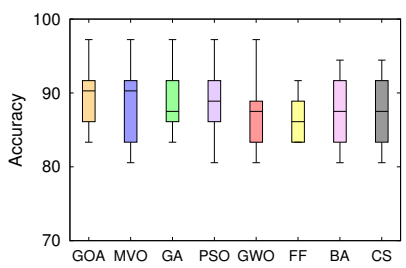

(q) Libras

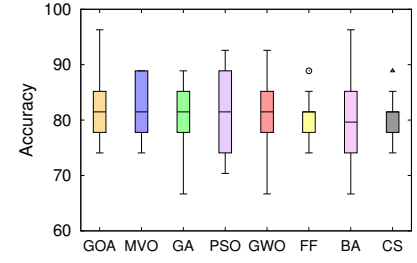

(c) Heart

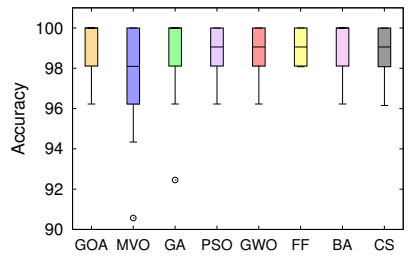

(f) Vowel

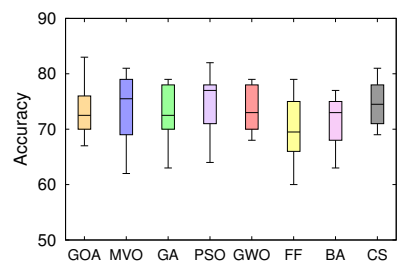

(i) German

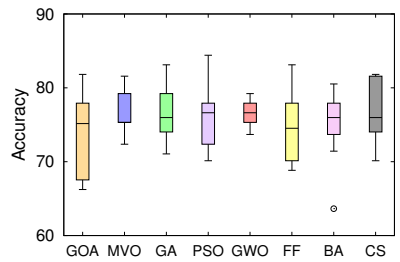

(l) diabetes

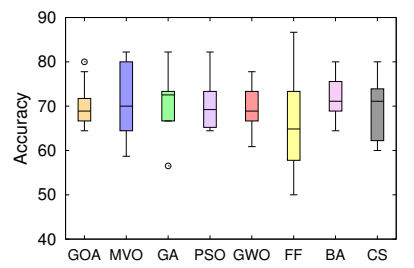

(o) Arrhythmia

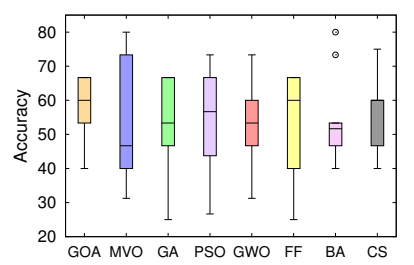

(r) Tae

Fig. 7: Box-plot charts for GOA and other algorithms based on 18 data sets after applying features selection. 
Table 7: Classification accuracy and standard deviation results of GOA and other approaches for all data sets after applying feature selection. (NFS stands for Number of selected features)

\begin{tabular}{|c|c|c|c|c|c|c|c|c|}
\hline \multirow{2}{*}{$\begin{array}{l}\text { Algorithm } \\
\text { Data }\end{array}$} & \multicolumn{2}{|c|}{ GOA } & \multicolumn{2}{|c|}{ MVO } & \multicolumn{2}{|c|}{ GA } & \multicolumn{2}{|c|}{$\mathrm{PSO}$} \\
\hline & $\operatorname{Avg} \pm \operatorname{Std}$ & $\mathrm{NSF}$ & $\operatorname{Avg} \pm \operatorname{Std}$ & NSF & $\operatorname{Avg} \pm$ Std & $\mathrm{NSF}$ & $\operatorname{Avg} \pm \operatorname{Std}$ & NSF \\
\hline Breast Cancer & $96.49 \pm 1.17$ & $6.70 \pm 1.19$ & $96.05 \pm 1.73$ & $5.10 \pm 1.45$ & $96.04 \pm 1.86$ & $6.40 \pm 1.56$ & $95.61 \pm 1.83$ & $6.40 \pm 1.43$ \\
\hline Wine & $97.77 \pm 2.72$ & $7.70 \pm 1.35$ & $97.19 \pm 3.75$ & $8.10 \pm 1.58$ & $97.19 \pm 2.81$ & $7.90 \pm 1.7$ & $95.55 \pm 3.33$ & $7.60 \pm 1.02$ \\
\hline Heart & $82.59 \pm 6.20$ & $7.10 \pm 1.22$ & $81.85 \pm 5.60$ & $8.60 \pm 1.69$ & $80.74 \pm 6.37$ & $6.50 \pm 2.38$ & $81.85 \pm 7.49$ & $8.40 \pm 1.20$ \\
\hline Parkinsons & $95.97 \pm 4.37$ & $12.90 \pm 1.92$ & $93.87 \pm 4.98$ & $12.10 \pm 2.84$ & $94.34 \pm 4.26$ & $8.70 \pm 1.79$ & $94.37 \pm 5.86$ & $10.40 \pm 2.11$ \\
\hline Sonar & $88.86 \pm 5.93$ & $31.80 \pm 3.54$ & $86.12 \pm 8.32$ & $29.90 \pm 2.43$ & $85.14 \pm 12.09$ & $21.90 \pm 4.97$ & $84.16 \pm 5.59$ & $29.70 \pm 2.97$ \\
\hline Vowel & $99.05 \pm 1.27$ & $7.40 \pm 0.92$ & $97.17 \pm 2.83$ & $8.20 \pm 0.75$ & $98.49 \pm 2.36$ & $8.20 \pm 0.60$ & $98.68 \pm 1.47$ & $8.00 \pm 1.26$ \\
\hline Spectft & $80.52 \pm 5.44$ & $19.80 \pm 3.16$ & $80.14 \pm 7.56$ & $20.20 \pm 3.54$ & $77.95 \pm 11.25$ & $14.30 \pm 2.00$ & $78.28 \pm 7.67$ & $20.40 \pm 4.86$ \\
\hline Ionosphere & $93.44 \pm 2.88$ & $16.80 \pm 2.27$ & $90.87 \pm 4.40$ & $15.50 \pm 3.44$ & $92.60 \pm 4.07$ & $14.00 \pm 3.49$ & $92.88 \pm 3.88$ & $18.70 \pm 1.79$ \\
\hline German & $73.40 \pm 4.45$ & $10.00 \pm 3.03$ & $73.90 \pm 6.42$ & $13.00 \pm 3.16$ & $72.60 \pm 4.82$ & $9.10 \pm 3.56$ & $74.80 \pm 5.65$ & $15.80 \pm 1.17$ \\
\hline Vehicle & $82.50 \pm 2.12$ & $11.40 \pm 1.28$ & $81.43 \pm 3.27$ & $13.00 \pm 2.10$ & $81.56 \pm 3.32$ & $11.70 \pm 2.19$ & $81.79 \pm 2.75$ & $13.20 \pm 1.25$ \\
\hline Australian & $85.07 \pm 5.27$ & $5.40 \pm 2.00$ & $86.81 \pm 5.04$ & $5.30 \pm 1.73$ & $84.64 \pm 5.39$ & $5.20 \pm 0.87$ & $84.06 \pm 4.44$ & $4.90 \pm 1.04$ \\
\hline Diabetes & $74.35 \pm 5.15$ & $4.10 \pm 1.58$ & $76.56 \pm 2.97$ & $4.80 \pm 1.78$ & $76.69 \pm 3.63$ & $6.30 \pm 1.62$ & $76.43 \pm 4.25$ & $5.40 \pm 1.28$ \\
\hline Liver & $67.51 \pm 8.05$ & $4.20 \pm 1.47$ & $62.87 \pm 8.34$ & $3.40 \pm 1.28$ & $66.83 \pm 10.10$ & $4.40 \pm 1.20$ & $66.18 \pm 1.28$ & $3.70 \pm 1.35$ \\
\hline Glass & $69.19 \pm 10.72$ & $5.20 \pm 1.17$ & $66.82 \pm 9.93$ & $5.00 \pm 0.89$ & $66.84 \pm 9.87$ & $5.20 \pm 1.40$ & $66.90 \pm 7.31$ & $5.10 \pm 0.94$ \\
\hline Arrhythmia & $70.35 \pm 4.75$ & $142.30 \pm 4.69$ & $70.80 \pm 7.84$ & $142.90 \pm 9.18$ & $70.83 \pm 6.44$ & $70.10 \pm 23.30$ & $70.14 \pm 5.39$ & $142.20 \pm 9.09$ \\
\hline Cleveland & $55.47 \pm 7.06$ & $6.90 \pm 2.12$ & $48.17 \pm 10.85$ & $6.30 \pm 1.79$ & $55.82 \pm 6.68$ & $5.30 \pm 2.00$ & $57.03 \pm 11.83$ & $6.20 \pm 1.66$ \\
\hline Libras & $89.44 \pm 4.44$ & $43.00 \pm 4.31$ & $88.33 \pm 5.09$ & $44.10 \pm 3.88$ & $88.61 \pm 4.20$ & $30.20 \pm 7.32$ & $89.17 \pm 4.56$ & $44.50 \pm 6.07$ \\
\hline TAE & $58.91 \pm 7.85$ & $3.40 \pm 1.02$ & $53.13 \pm 17.12$ & $3.90 \pm 0.94$ & $51.83 \pm 14.54$ & $4.60 \pm 0.49$ & $54.38 \pm 14.11$ & $4.50 \pm 0.67$ \\
\hline \multirow[t]{2}{*}{ Algorithm } & \multicolumn{2}{|c|}{ GWO } & \multicolumn{2}{|c|}{ FF } & \multicolumn{2}{|c|}{$\mathrm{BA}$} & \multicolumn{2}{|c|}{ CS } \\
\hline & $\operatorname{Avg} \pm$ Std & NSF & $\operatorname{Avg} \pm$ Std & NSF & $\operatorname{Avg} \pm$ Std & NSF & $\operatorname{Avg} \pm$ Std & NSF \\
\hline Breast Cancer & $95.89 \pm 2.86$ & $5.40 \pm 1.62$ & $96.33 \pm 2.57$ & $4.70 \pm 1.27$ & $96.20 \pm 1.74$ & $5.60 \pm 1.50$ & $96.48 \pm 1.64$ & $5.90 \pm 1.45$ \\
\hline Wine & $97.22 \pm 2.78$ & $7.10 \pm 1.45$ & $96.11 \pm 4.34$ & $7.10 \pm 2.02$ & $94.41 \pm 6.57$ & $7.00 \pm 1.18$ & $96.57 \pm 3.85$ & $8.10 \pm 1.04$ \\
\hline Heart & $80.74 \pm 7.55$ & $6.90 \pm 1.81$ & $81.11 \pm 3.87$ & $6.00 \pm 2.53$ & $80.37 \pm 7.95$ & $7.00 \pm 2.45$ & $81.11 \pm 3.87$ & $8.40 \pm 1.02$ \\
\hline Parkinsons & $95.92 \pm 5.85$ & $9.10 \pm 2.47$ & $92.37 \pm 6.83$ & $11.30 \pm 1.10$ & $92.29 \pm 4.67$ & $12.70 \pm 1.68$ & $95.37 \pm 4.30$ & $10.90 \pm 2.21$ \\
\hline Sonar & $88.00 \pm 5.70$ & $19.40 \pm 1.96$ & $86.55 \pm 8.24$ & $28.60 \pm 4.29$ & $86.09 \pm 5.34$ & $28.10 \pm 4.41$ & $85.64 \pm 7.91$ & $29.7 \pm 3.58$ \\
\hline Vowel & $98.86 \pm 1.25$ & $8.50 \pm 1.02$ & $99.05 \pm 0.95$ & $8.30 \pm 0.64$ & $99.05 \pm 1.27$ & $8.30 \pm 1.00$ & $98.67 \pm 1.49$ & $8.40 \pm 0.92$ \\
\hline Spectft & $76.38 \pm 5.68$ & $16.10 \pm 3.27$ & $77.46 \pm 10.48$ & $19.80 \pm 2.32$ & $77.18 \pm 8.94$ & $18.90 \pm 2.70$ & $77.12 \pm 9.46$ & $18.80 \pm 2.89$ \\
\hline Ionosphere & $92.59 \pm 3.67$ & $13.70 \pm 2.10$ & $90.88 \pm 5.24$ & $14.80 \pm 3.28$ & $90.88 \pm 4.75$ & $16.70 \pm 2.90$ & $92.89 \pm 5.87$ & $16.70 \pm 4.15$ \\
\hline German & $73.50 \pm 3.91$ & $12.30 \pm 1.55$ & $70.20 \pm 5.36$ & $10.40 \pm 2.20$ & $71.70 \pm 4.50$ & $11.90 \pm 1.51$ & $74.70 \pm 3.77$ & $15.40 \pm 2.69$ \\
\hline Vehicle & $81.32 \pm 5.68$ & $11.40 \pm 1.11$ & $81.68 \pm 3.36$ & $11.80 \pm 1.17$ & $80.14 \pm 6.55$ & $11.40 \pm 2.00$ & $81.67 \pm 4.31$ & $13.20 \pm 1.66$ \\
\hline Australian & $85.36 \pm 4.07$ & $5.10 \pm 0.70$ & $85.07 \pm 3.55$ & $3.60 \pm 0.49$ & $84.35 \pm 4.09$ & $4.90 \pm 0.94$ & $84.49 \pm 6.12$ & $7.40 \pm 2.29$ \\
\hline Diabetes & $76.69 \pm 1.84$ & $5.30 \pm 1.19$ & $74.87 \pm 4.50$ & $3.30 \pm 1.42$ & $75.13 \pm 4.58$ & $4.80 \pm 1.47$ & $76.70 \pm 4.20$ & $6.10 \pm 1.04$ \\
\hline Liver & $62.01 \pm 7.04$ & $3.40 \pm 1.56$ & $66.43 \pm 7.10$ & $2.90 \pm 1.30$ & $62.92 \pm 6.88$ & $4.00 \pm 1.55$ & $68.11 \pm 7.26$ & $5.40 \pm 0.66$ \\
\hline Glass & $63.57 \pm 8.10$ & $4.30 \pm 0.64$ & $67.27 \pm 7.82$ & $4.60 \pm 1.11$ & $68.29 \pm 10.96$ & $4.80 \pm 1.33$ & $68.64 \pm 13.13$ & $5.00 \pm 1.18$ \\
\hline Arrhythmia & $69.71 \pm 4.67$ & $67.00 \pm 14.02$ & $66.41 \pm 10.49$ & $137.70 \pm 6.21$ & $71.68 \pm 4.64$ & $142.30 \pm 6.77$ & $69.92 \pm 6.59$ & $140.70 \pm 5.2$ \\
\hline Cleveland & $56.10 \pm 8.49$ & $6.20 \pm 2.18$ & $55.42 \pm 10.30$ & $5.20 \pm 1.99$ & $55.80 \pm 9.60$ & $5.60 \pm 1.50$ & $58.09 \pm 7.70$ & $7.10 \pm 1.51$ \\
\hline Libras & $87.22 \pm 5.30$ & $30.40 \pm 4.76$ & $86.39 \pm 3.15$ & $44.40 \pm 5.06$ & $87.22 \pm 4.51$ & $43.60 \pm 3.98$ & $87.5 \pm 4.167$ & $43.60 \pm 4.15$ \\
\hline TAE & $52.46 \pm 11.70$ & $3.70 \pm 0.90$ & $52.50 \pm 16.18$ & $4.40 \pm 0.80$ & $53.67 \pm 12.51$ & $3.90 \pm 0.94$ & $56.83 \pm 9.84$ & $4.00 \pm 0.77$ \\
\hline
\end{tabular}

high exploratory behaviour and this was the main reason of its success it this work. As per the results and No Free Lunch theorem, we state that this algorithm is worthy of use in the field of feature selection to assist classifier in finding a suitable set of features. It has the potential to leverage the whole classification process.

\section{Conclusions}

This work presents a novel hybrid approach for optimising SVM based on the Grasshopper Optimisation Algorithm (GOA). The proposed approach is able to tune the parameters of the SVM kernel and at the same time can find the minimal and optimal subset of the model features. This study shows that optimising SVM classifier by finding suitable features and the optimal kernel parameters simultaneously lead to better overall classification accuracy. The experimental results on the benchmark data sets revealed the effectiveness of the GOA on enhancing the SVM classifier accuracy. The GOA outperforms other optimisers such as GA, MVO, GWO, PSO, Firefly, BA, and Cuckoo search in most of the data sets in terms of classification accuracy.

For future work, the application of the proposed GOA-SVM model to other real word problems can be investigated and applied. For example, the efficacy of the proposed model could be employed to tackle data mining problems in different domains such as real business problems in organizational environments. Furthermore, the performance of the model can be investigated on larger scale problems. 
Table 8: P-values of the Wilcoxon test of GOA classification results vs other algorithms after applying features selection ( $\mathrm{p} \geq 0.05$ are underlined).

\begin{tabular}{|c|c|c|c|c|c|c|c|}
\hline data set/Algorithm & MVO & GA & PSO & GWO & $\mathrm{FF}$ & $\mathrm{BA}$ & CS \\
\hline Breast Cancer & $2.56 \mathrm{E}-02$ & $4.21 \mathrm{E}-03$ & $5.23 \mathrm{E}-07$ & $2.06 \mathrm{E}-01$ & $5.37 \mathrm{E}-01$ & $6.18 \mathrm{E}-01$ & $9.02 \mathrm{E}-01$ \\
\hline Wine & $\underline{2.62 \mathrm{E}-01}$ & $\underline{5.14 \mathrm{E}-02}$ & $2.73 \mathrm{E}-06$ & $1.57 \mathrm{E}-01$ & $1.34 \mathrm{E}-02$ & $1.83 \mathrm{E}-05$ & $1.34 \mathrm{E}-02$ \\
\hline Heart & 7.10E-01 & $4.54 \mathrm{E}-01$ & $4.59 \mathrm{E}-01$ & $3.85 \mathrm{E}-01$ & $3.13 \mathrm{E}-01$ & $2.55 \mathrm{E}-02$ & $3.13 \mathrm{E}-01$ \\
\hline Parkinsons & $5.97 \mathrm{E}-04$ & $9.02 \mathrm{E}-04$ & $9.38 \mathrm{E}-02$ & $1.27 \mathrm{E}-14$ & $3.19 \mathrm{E}-05$ & $2.66 \mathrm{E}-10$ & $1.22 \mathrm{E}-01$ \\
\hline Sonar & $2.57 \mathrm{E}-02$ & $6.40 \mathrm{E}-02$ & $8.57 \mathrm{E}-09$ & 2.13E-01 & $3.75 \mathrm{E}-01$ & $1.55 \mathrm{E}-04$ & $5.58 \mathrm{E}-03$ \\
\hline Vowel & $1.23 \mathrm{E}-07$ & $6.78 \mathrm{E}-01$ & $1.76 \mathrm{E}-01$ & $2.80 \mathrm{E}-01$ & $6.84 \mathrm{E}-01$ & $1.00 \mathrm{E}+00$ & $\underline{5.96 \mathrm{E}-02}$ \\
\hline Spectft & $8.06 \mathrm{E}-01$ & $6.22 \mathrm{E}-01$ & $1.39 \mathrm{E}-01$ & $1.24 \mathrm{E}-04$ & $1.76 \mathrm{E}-01$ & $\overline{1.40 \mathrm{E}-03}$ & $3.20 \mathrm{E}-03$ \\
\hline Ionosphere & $1.45 \mathrm{E}-06$ & $1.31 \mathrm{E}-01$ & $1.69 \mathrm{E}-01$ & $2.12 \mathrm{E}-01$ & $1.30 \mathrm{E}-05$ & $1.76 \mathrm{E}-03$ & $9.02 \mathrm{E}-01$ \\
\hline German & $\underline{5.00 \mathrm{E}-02}$ & 9.03E-01 & $4.78 \mathrm{E}-03$ & $3.26 \mathrm{E}-01$ & $1.76 \mathrm{E}-05$ & $2.19 \mathrm{E}-01$ & $2.73 \mathrm{E}-02$ \\
\hline Vehicle & $\overline{2.69 \mathrm{E}-02}$ & $2.20 \mathrm{E}-01$ & $2.18 \mathrm{E}-01$ & $1.42 \mathrm{E}-02$ & $3.21 \mathrm{E}-03$ & $\overline{3.71 \mathrm{E}-02}$ & 7.14E-01 \\
\hline Australian & $\underline{6.50 \mathrm{E}-02}$ & $1.10 \mathrm{E}-01$ & $\underline{1.40 \mathrm{E}-01}$ & $\underline{2.66 \mathrm{E}-01}$ & $\underline{7.12 \mathrm{E}-01}$ & $\underline{4.60 \mathrm{E}-01}$ & $\overline{7.11 \mathrm{E}-01}$ \\
\hline Diabetes & $\overline{4.50 \mathrm{E}-03}$ & $\overline{9.93 \mathrm{E}-03}$ & $\overline{1.35 \mathrm{E}-02}$ & $\overline{9.74 \mathrm{E}-03}$ & $\overline{3.90 \mathrm{E}-01}$ & $\overline{3.91 \mathrm{E}-01}$ & $\overline{9.99 \mathrm{E}-03}$ \\
\hline Liver & $9.08 \mathrm{E}-04$ & $\underline{8.07 \mathrm{E}-01}$ & $2.20 \mathrm{E}-01$ & $2.06 \mathrm{E}-34$ & $3.25 \mathrm{E}-01$ & $3.04 \mathrm{E}-05$ & $\underline{3.26 \mathrm{E}-01}$ \\
\hline Glass & $\underline{3.28 \mathrm{E}-01}$ & $\overline{6.24 \mathrm{E}-01}$ & $\underline{4.63 \mathrm{E}-01}$ & $3.13 \mathrm{E}-05$ & $8.07 \mathrm{E}-01$ & $1.00 \mathrm{E}+00$ & $1.00 \mathrm{E}+00$ \\
\hline Arrhythmia & $1.00 \mathrm{E}+00$ & $3.63 \mathrm{E}-02$ & $\underline{5.40 \mathrm{E}-01}$ & $2.21 \mathrm{E}-01$ & $2.35 \mathrm{E}-04$ & $1.36 \mathrm{E}-02$ & $\underline{6.24 \mathrm{E}-01}$ \\
\hline Cleveland & $\overline{3.04 \mathrm{E}-06}$ & $2.67 \mathrm{E}-01$ & $8.07 \mathrm{E}-01$ & $7.13 \mathrm{E}-01$ & $5.39 \mathrm{E}-01$ & $9.03 \mathrm{E}-01$ & $9.76 \mathrm{E}-03$ \\
\hline Libras & 7.77E-02 & $1.68 \mathrm{E}-01$ & $8.04 \mathrm{E}-01$ & $8.36 \mathrm{E}-04$ & $9.03 \mathrm{E}-07$ & $1.91 \mathrm{E}-03$ & $4.22 \mathrm{E}-03$ \\
\hline TAE & $3.46 \mathrm{E}-04$ & $1.12 \mathrm{E}-03$ & $1.71 \mathrm{E}-01$ & $4.27 \mathrm{E}-06$ & $\underline{3.11 \mathrm{E}-01}$ & $7.29 \mathrm{E}-07$ & $2.37 \mathrm{E}-02$ \\
\hline
\end{tabular}

Table 9: Summary statistical results on a variety of measures (with feature selection). The table values represent the number of data sets each algorithm won/losses/ties on a variety of measures along with their rank using F-test.

\begin{tabular}{|c|c|c|c|c|c|c|c|c|c|c|c|c|c|c|c|c|c|c|c|c|c|c|c|c|}
\hline \multirow[t]{2}{*}{ Algorithm } & \multicolumn{3}{|c|}{ GOA } & \multicolumn{3}{|c|}{ MVO } & \multicolumn{3}{|c|}{ GA } & \multicolumn{3}{|c|}{ PSO } & \multicolumn{3}{|c|}{ GWO } & \multicolumn{3}{|c|}{ FF } & \multicolumn{3}{|c|}{$\mathrm{BA}$} & \multicolumn{3}{|c|}{ CS } \\
\hline & W & $\mathrm{T}$ & $\mathrm{L}$ & W & $\mathrm{T}$ & $\mathrm{L}$ & W & $\mathrm{T}$ & $\mathrm{L}$ & W & $\mathrm{T}$ & $\mathrm{L}$ & W & $\mathrm{T}$ & $\mathrm{L}$ & W & $\mathrm{T}$ & $\mathrm{L}$ & W & $\mathrm{T}$ & $\mathrm{L}$ & W & $\mathrm{T}$ & L \\
\hline Accur & 12 & 1 & 7 & 1 & 0 & 17 & 0 & 0 & 18 & 1 & 0 & 17 & 0 & 0 & 18 & 0 & 1 & 17 & 1 & 1 & 16 & 1 & 0 & 17 \\
\hline Featu & 2 & 1 & 15 & 0 & 0 & 18 & 3 & 0 & 15 & 0 & 0 & 18 & 5 & 1 & 12 & 7 & 0 & 11 & 0 & 1 & 17 & 0 & 0 & 18 \\
\hline P-value & - & - & - & 1 & 8 & 9 & 2 & 12 & 4 & 1 & 11 & 6 & 1 & 10 & 7 & 0 & 11 & 7 & 1 & 7 & 10 & 2 & 9 & 7 \\
\hline Rank & & 2.31 & & & 4.83 & & & 4.86 & & & 4.36 & & & 5.08 & & & 5.31 & & & 5.61 & & & 3.64 & \\
\hline
\end{tabular}

\section{Compliance with Ethical Standards}

Conflict of Interests The authors declare that they have no conflict of interest.

Informed Consent All procedures followed were in accordance with the ethical standards of the responsible committee on human experimentation (institutional and national) and with the Helsinki Declaration of 1975, as revised in 2008 (5).

Human and Animal Rights This article does not contain any studies with human or animal subjects performed by any of the authors.

\section{Funding}

This research received no specific grant from any funding agency in public, commercial, or not-for-profit sectors.

Acknowledgements The authors would like to thank Dr. Simon Andrews from Babraham Institute, Cambridge, UK for thoroughly proof-reading this paper.

\section{References}

1. Aljarah, I., Faris, H., Mirjalili, S.: Optimizing connection weights in neural networks using the whale optimization algorithm. Soft Computing pp. 1-15 (2016)

2. Arana-Daniel, N., Gallegos, A.A., López-Franco, C., Alanís, A.Y., Morales, J., López-Franco, A.: Support vector machines trained with evolutionary algorithms employing kernel adatron for large scale classification of pro- 
Table 10: Key to comparative methods.

\begin{tabular}{|c|c|c|c|c|}
\hline Key & Method name & $\begin{array}{l}\text { Population } \\
\text { x Iteration }\end{array}$ & $\begin{array}{l}\text { data set } \\
\text { Number }\end{array}$ & Reference \\
\hline GA-SVM & $\begin{array}{l}\text { Genetic algorithm based feature selection and } \\
\text { parameters optimization for support vector } \\
\text { machines. }\end{array}$ & $500 \times 600$ & 11 & {$[16]$} \\
\hline MVO-SVM & $\begin{array}{l}\text { A multi-verse optimizer approach for feature } \\
\text { selection and optimizing SVM parameters based on } \\
\text { a robust system architecture }\end{array}$ & $50 \times 30$ & 10 & {$[12]$} \\
\hline PSO-SVM & $\begin{array}{l}\text { Particle swarm optimization for parameter } \\
\text { determination and feature selection of support } \\
\text { vector machines. }\end{array}$ & $8 \times 250$ & 17 & {$[22]$} \\
\hline GSA-SVM & $\begin{array}{l}\text { Gravitational search algorithm and support vector } \\
\text { machines hybrid system for classification of binary } \\
\text { problems. }\end{array}$ & $5 \times 20$ & 8 & {$[20]$} \\
\hline Grid-SVM & Grid search & - & 17 & {$[22]$} \\
\hline GOA-SVM & $\begin{array}{l}\text { Grasshopper Optimisation Algorithm for Feature } \\
\text { selection and parameters optimisation support } \\
\text { vector machines }\end{array}$ & $100 \times 200$ & 18 & Our \\
\hline
\end{tabular}

Table 11: Classification accuracy comparison between GOA and different methods in the literature.

\begin{tabular}{llccccc}
\hline data set & GOA & Grid search & MVO & GA & PSO & GSA \\
\hline Wine & $\mathbf{1 0 0 . 0 0}$ & 96.60 & $\mathbf{1 0 0 . 0 0}$ & - & $\mathbf{1 0 0 . 0 0}$ & - \\
Ionosphere & $\mathbf{9 9 . 4 3}$ & 93.08 & 99.15 & 98.56 & 99.01 & 96.01 \\
Iris & $\mathbf{1 0 0 . 0 0}$ & 96.00 & - & $\mathbf{1 0 0 . 0 0}$ & 99.20 & - \\
Pima & 81.52 & 76.69 & - & 81.50 & 82.68 & $\mathbf{9 4 . 0 9}$ \\
Vehicle & $\mathbf{9 0 . 1 8}$ & 84.28 & 89.00 & 84.06 & 89.83 & - \\
Vowel & $\mathbf{1 0 0 . 0 0}$ & 98.91 & $\mathbf{1 0 0 . 0 0}$ & 99.30 & 100.00 & - \\
Australian & 91.45 & 84.84 & - & 88.10 & 91.03 & $\mathbf{9 5 . 7 1}$ \\
Breast & $\mathbf{9 9 . 2 7}$ & 96.64 & $\mathbf{9 9 . 2 7}$ & 96.19 & 99.18 & 95.57 \\
cancer & & & & & & \\
German & 82.20 & 75.30 & 79.20 & 85.60 & 81.62 & $\mathbf{9 4 . 5 1}$ \\
Sonar & 98.07 & 87.90 & $\mathbf{9 9 . 0 2}$ & 98.00 & 96.26 & - \\
Parkinsons & $\mathbf{1 0 0 . 0 0}$ & - & 99.50 & - & - & - \\
Spectf & $\mathbf{9 6 . 2 5}$ & - & 95.91 & - & - & - \\
\hline
\end{tabular}

- The results of this data set are not available.

tein structures. Evolutionary Bioinformatics Online 12, 285 (2016)

3. Babaoğlu, I., Fındık, O., Bayrak, M.: Effects of principle component analysis on assessment of coronary artery diseases using support vector machine. Expert Systems with Applications 37(3), 2182-2185 (2010)

4. Bao, Y., Hu, Z., Xiong, T.: A pso and pattern search based memetic algorithm for svms parameters optimization. Neurocomputing 117, 98-106 (2013)

5. Beyer, K., Goldstein, J., Ramakrishnan, R., Shaft, U.: When is nearest neighbor meaningful? In: International conference on database theory, pp. 217-235. Springer
(1999)

6. Blondin, J., Saad, A.: Metaheuristic techniques for support vector machine model selection. In: Hybrid Intelligent Systems (HIS), 2010 10th International Conference on, pp. 197-200. IEEE (2010)

7. Boser, B.E., Guyon, I.M., Vapnik, V.N.: A training algorithm for optimal margin classifiers. In: Proceedings of the fifth annual workshop on Computational learning theory, pp. 144-152. ACM (1992)

8. Bouraoui, A., Jamoussi, S., BenAyed, Y.: A multiobjective genetic algorithm for simultaneous model and feature selection for support vector machines. Artificial 
Intelligence Review pp. 1-21 (2017)

9. Chang, C.C., Lin, C.J.: Libsvm: a library for support vector machines. ACM Transactions on Intelligent Systems and Technology (TIST) 2(3), 27 (2011)

10. Cortes, C., Vapnik, V.: Support-vector networks. Machine learning 20(3), 273-297 (1995)

11. Eswaramoorthy, S., Eswaramoorthy, S., Sivakumaran, N., Sivakumaran, N., Sekaran, S., Sekaran, S.: Grey wolf optimization based parameter selection for support vector machines. COMPEL-The international journal for computation and mathematics in electrical and electronic engineering 35(5), 1513-1523 (2016)

12. Faris, H., Hassonah, M.A., AlaM, A.Z., Mirjalili, S., Aljarah, I.: A multi-verse optimizer approach for feature selection and optimizing svm parameters based on a robust system architecture. Neural Computing and Applications pp. $1-15$

13. Gepperth, A., Karaoguz, C.: A bio-inspired incremental learning architecture for applied perceptual problems. Cognitive Computation 8(5), 924-934 (2016)

14. Goldberg, D.E.: Genetic Algorithms in Search, Optimization and Machine Learning, 1st edn. Addison-Wesley Longman Publishing Co., Inc., Boston, MA, USA (1989)

15. Huang, C.L.: Aco-based hybrid classification system with feature subset selection and model parameters optimization. Neurocomputing 73(1), 438-448 (2009)

16. Huang, C.L., Wang, C.J.: A ga-based feature selection and parameters optimizationfor support vector machines. Expert Systems with applications 31(2), 231-240 (2006)

17. James, G., Witten, D., Hastie, T., Tibshirani, R.: An introduction to statistical learning, vol. 6. Springer (2013)

18. Kanevski, M., Pozdnukhov, A., Timonin, V.: Machine learning algorithms for geospatial data. applications and software tools (2008)

19. Kohavi, R., John, G.H.: Wrappers for feature subset selection. Artificial intelligence 97(1), 273-324 (1997)

20. Li, C., An, X., Li, R.: A chaos embedded gsa-svm hybrid system for classification. Neural Computing and Applications 26(3), 713-721 (2015)

21. Lichman, M.: UCI machine learning repository (2013). URL http://archive.ics.uci.edu/ml

22. Lin, S.W., Ying, K.C., Chen, S.C., Lee, Z.J.: Particle swarm optimization for parameter determination and feature selection of support vector machines. Expert systems with applications 35(4), 1817-1824 (2008)

23. Liu, X., Zeng, Y., Zhang, T., Xu, B.: Parallel brain simulator: A multi-scale and parallel brain-inspired neural network modeling and simulation platform. Cognitive Computation 8(5), 967-981 (2016)

24. Liu, Y., Wang, G., Chen, H., Dong, H., Zhu, X., Wang, S.: An improved particle swarm optimization for feature selection. Journal of Bionic Engineering 8(2), 191-200 (2011)

25. Mirjalili, S., Mirjalili, S.M., Hatamlou, A.: Multi-verse optimizer: a nature-inspired algorithm for global optimization. Neural Computing and Applications 27(2), 495-513 (2016)

26. Mirjalili, S., Mirjalili, S.M., Lewis, A.: Grey wolf optimizer. Advances in Engineering Software 69, 46-61 (2014)

27. Mirjalili, S.Z., Mirjalili, S., Saremi, S., Faris, H., Aljarah, I.: Grasshopper optimization algorithm for multiobjective optimization problems. Applied Intelligence pp. $1-16$ (2017)

28. Nguyen, M.H., De la Torre, F.: Optimal feature selection for support vector machines. Pattern recognition 43(3), 584-591 (2010)
29. Perdisci, R., Gu, G., Lee, W.: Using an ensemble of oneclass svm classifiers to harden payload-based anomaly detection systems. In: Data Mining, 2006. ICDM'06. Sixth International Conference on, pp. 488-498. IEEE (2006)

30. Puntura, A., Theera-Umpon, N., Auephanwiriyakul, S.: Optimizing support vector machine parameters using cuckoo search algorithm via cross validation. In: Control System, Computing and Engineering (ICCSCE), 2016 6th IEEE International Conference on, pp. 102-107. IEEE (2016)

31. Rodan, A., Faris, H.: Credit risk evaluation using cycle reservoir neural networks with support vector machines readout. In: Asian Conference on Intelligent Information and Database Systems, pp. 595-604. Springer (2016)

32. Rodan, A., Faris, H., Alsakran, J., Al-Kadi, O.: A support vector machine approach for churn prediction in telecom industry. International Information Institute (Tokyo). Information 17(8), 3961 (2014)

33. Samadzadegan, F., Soleymani, A., Abbaspour, R.A.: Evaluation of genetic algorithms for tuning svm parameters in multi-class problems. In: Computational Intelligence and Informatics (CINTI), 2010 11th International Symposium on, pp. 323-328. IEEE (2010)

34. Saremi, S., Mirjalili, S., Lewis, A.: Grasshopper optimisation algorithm: Theory and application. Advances in Engineering Software 105, 30-47 (2017)

35. Sheta, A., Ahmed, S.E., Faris, H.: A comparison between regression, artificial neural networks and support vector machines for predicting stock market index. International Journal of Advanced Research in Artificial Intelligence (IJARAI) 4(7), 55-63 (2015)

36. Sotiris, V.A., Peter, W.T., Pecht, M.G.: Anomaly detection through a bayesian support vector machine. IEEE Transactions on Reliability 59(2), 277-286 (2010)

37. Staelin, C.: Parameter selection for support vector machines. Hewlett-Packard Company, Tech. Rep. HPL2002-354R1 (2003)

38. Takeuchi, K., Collier, N.: Bio-medical entity extraction using support vector machines. Artificial Intelligence in Medicine 33(2), 125-137 (2005)

39. Tanveer, M.: Robust and sparse linear programming twin support vector machines. Cognitive Computation 7(1), 137-149 (2015)

40. Tuba, E., Mrkela, L., Tuba, M.: Support vector machine parameter tuning using firefly algorithm. In: Radioelektronika (RADIOELEKTRONIKA), 2016 26th International Conference, pp. 413-418. IEEE (2016)

41. Tuba, E., Tuba, M., Simian, D.: Adjusted bat algorithm for tuning of support vector machine parameters. In: Evolutionary Computation (CEC), 2016 IEEE Congress on, pp. 2225-2232. IEEE (2016)

42. Vapnik, V.: An overview of statistical learning theory. IEEE Transactions on Neural Networks 5, 988-999 (1999)

43. Vapnik, V.: The nature of statistical learning theory. Springer Science \& Business Media (2013)

44. Weston, J., Mukherjee, S., Chapelle, O., Pontil, M., Poggio, T., Vapnik, V.: Feature selection for svms. In: Proceedings of the 13th International Conference on Neural Information Processing Systems, pp. 647-653. MIT Press (2000)

45. Wolpert, D.H., Macready, W.G.: No free lunch theorems for optimization. IEEE transactions on evolutionary computation 1(1), 67-82 (1997)

46. Wu, T., Yao, M., Yang, J.: Dolphin swarm extreme learning machine. Cognitive Computation 9(2), 275-284 (2017) 
47. Xin, J., Chen, G., Hai, Y.: A particle swarm optimizer with multi-stage linearly-decreasing inertia weight. In: Computational Sciences and Optimization, 2009. CSO 2009. International Joint Conference on, vol. 1, pp. 505508. IEEE (2009)

48. Xu, Y., Guo, R., Wang, L.: A twin multi-class classification support vector machine. Cognitive Computation 5(4), 580-588 (2013)

49. Yang, J., Gong, L., Tang, Y., Yan, J., He, H., Zhang, L., Li, G.: An improved svm-based cognitive diagnosis algorithm for operation states of distribution grid. Cognitive Computation 7(5), 582-593 (2015)

50. Yang, X.S., Deb, S.: Cuckoo search via lévy flights. In: Nature \& Biologically Inspired Computing, 2009. NaBIC 2009. World Congress on, pp. 210-214. IEEE (2009)

51. Yang, X.S., Deb, S., Fong, S.: Accelerated particle swarm optimization and support vector machine for business optimization and applications. In: International Conference on Networked Digital Technologies, pp. 53-66. Springer (2011)

52. Yang, X.S., He, X.: Firefly algorithm: recent advances and applications. International Journal of Swarm Intel- ligence 1(1), 36-50 (2013)

53. Yang, X.S., Hossein Gandomi, A.: Bat algorithm: a novel approach for global engineering optimization. Engineering Computations 29(5), 464-483 (2012)

54. Yin, S., Zhu, X., Jing, C.: Fault detection based on a robust one class support vector machine. Neurocomputing 145, 263-268 (2014)

55. Zhang, H., Berg, A.C., Maire, M., Malik, J.: Svm-knn: Discriminative nearest neighbor classification for visual category recognition. In: Computer Vision and Pattern Recognition, 2006 IEEE Computer Society Conference on, vol. 2, pp. 2126-2136. IEEE (2006)

56. Zhang, J., Tittel, F.K., Gong, L., Lewicki, R., Griffin, R.J., Jiang, W., Jiang, B., Li, M.: Support vector machine modeling using particle swarm optimization approach for the retrieval of atmospheric ammonia concentrations. Environmental Modeling \& Assessment 21(4), 531-546 (2016)

57. Zhang, X., Chen, X., He, Z.: An aco-based algorithm for parameter optimization of support vector machines. Expert Systems with Applications 37(9), 6618-6628 (2010) 